\begin{tabular}{ccr} 
FOLIA & ENTOMOLOGICA & HUNGARICA \\
& ROVARTANI KÖZLEMÉNYEK & \\
Volume 81 & 2020 & pp. $43-72$ \\
\hline
\end{tabular}

\title{
Longhorn beetles in the entomological collections of the Republic of Moldova (Coleoptera: Cerambycidae)
}

\author{
Svetlana BACAL ${ }^{1}$, Daniela Burduja ${ }^{1}$, Galina Buşmachiu ${ }^{1 *}$, Cristina Cebotari ${ }^{1} \&$ \\ Ottó MERKL ${ }^{2}$ \\ ${ }^{1}$ Institute of Zoology, Academiei str 1, MD-2028 Chişinău, Republic of Moldova. \\ E-mails:svetabacal@yahoo.com,dana.virlan@mail.ru,bushmakiu@yahoo.com, \\ cebotari15251@gmail.com \\ ${ }^{2}$ Hungarian Natural History Museum, Department of Zoology, H-1088 Budapest, Baross utca 13, \\ Hungary.E-mail:merkl.otto@nhmus.hu
}

\begin{abstract}
The first inventory of Cerambycidae species kept in the collections of the Republic of Moldova is presented. A total of 121 species belonging to 60 genera and 6 subfamilies is listed, of them 5 are cited for the first time in the country. The specimens were collected between 1912 and 2019 and are kept in four entomological collections in the capital city Chişinau: the Museum of Entomology, the Institute of Zoology, the Museum of the Institute of Genetics, Physiology and Plant Protection, the National Museum of Ethnography and Natural History and the Museum of the State University of Moldova. The list of Cerambycidae species known to occur in Moldova has been expanded to 148 species.
\end{abstract}

Key words - insect, museum, list of species, new records

\section{INTRODUCTION}

The family of longhorn beetles (Coleoptera: Cerambycidae) is a diverse and economically important group of insects, with a number of species estimated at 35000 (Nearns 2013). Cerambycidae are found on all continents except Antarctica, and comprise one of the largest beetle families.

The oldest known museum specimen of Cerambycidae from present-day Republic of Moldova, an Agapanthia dabli (Richter, 1821), was collected in July 1912. It is part of the oldest insect collection built up between 1912 and 1939 by N. N. Zubowsky and is kept in the first public museum of Bessarabia, now the National Museum of Ethnography and Natural History, founded in 1889.

* Corresponding author. 
The second largest collection of insects, including several Cerambycidae, were assembled by R. Stepanov during 1953-1979, and is housed in the Museum of Entomology, the Institute of Zoology. Besides the insects collected by Stepanov himself, the Museum of Entomology holds longhorn beetles collected by S. T,epala (in 1954), S. Plugaru (between 1954-1978), V. Ostaficiuc (between 1960-1977) and S. Bacal (between 2006-2019).

Specimens of harmful insects, including several Cerambycidae, are housed in the Museum of the Institute of Genetics, Physiology and Plant Protection. These were collected by O. Crijanovski (between 1974-1976), L. Egorov (between 1986-1989), A. Lobanov (in 1986), V. A. Mațiuc (in 1986) and others.

Another large zoological collection, with insects included, is kept in the Museum of State University of Moldova, in which the Cerambycidae material has a special place in the museum exhibitions. The specimens were collected mostly by students and employees of the university in the summer internships. Unfortunately, not all specimens are correctly labelled; especially the year of collection is often missing. This insect collection is part of the educational material of the Faculty of Biology.

The present paper is based exclusively on the specimens collected in the present-day territory of the country. Specimens collected abroad will be the subject of other publications.

The aim of this study was to compile the first inventory of the Cerambycidae specimens kept in the museums of Chişinău, that have an undeniable value not only for the Republic of Moldova but also for researchers around the world.

\section{MATERIAL AND METHODS}

The specimens of Cerambycidae accumulated between 1912 and 2019 are kept in four collections in the Republic of Moldova, as listed in the Introduction. The specimens were collected from the whole territory of the country, in 20 districts and 53 localities from the Central, Southern and Northern Regions.

The Central Region includes three municipalities, 10 districts and 37 localities: Chişinău municipality with the towns Durleşti, Codru and Vadul-lui-Vodă; Bender municipality;

Tiraspol municipality;

Anenii Noi district and the localities Calfa, Cebanovca, Hîrbovăț and Socoleni;

Călăraşi district and localities Bahmut, Bularda, Leordoaia and Tuzara;

Criuleni district and localities Dubăsarii Vechi, Hruşova and Onițcani;

Dubăsari district and locality Goian;

Hânceşti district and localities Leuşeni, Logăneşti and Minjir;

Ialoveni district and localities Dănceni and Suruceni;

Nisporeni district and locality Cioreşti; 
Orhei district and localities Curchi, Dişcova, Ivancea, Trebujeni and Vatici; Străşeni district and localities Lozova (Scientific Reserve Codrii) and Codreanca (in the past Cobîlca);

Ungheni district and localities Corneşti, Rădenii Vechi (Reserve Plaiul Fagului) and Sculeni.

The Southern Region includes four districts, one territorial unit and eleven localities:

Cantemir district and locality Cociulia;

Căuşeni district and localities Chițcani and Hagimus;

Cimişlia district and locality Zloți;

Ştefan Vodă district and localities Olăneşti, Tudora and Palanca;

Territorial Unit of Gagauzia and town Comrat.

The Northern Region includes two districts and tree localities:

Briceni district and localities Caracuşeni and Trebisăuți;

Şoldăneşti district and locality Cobîlea.

The specimens were examined under a binocular MBS 10 .

Abbreviations - The museums mentioned in the text are referred to by abbreviations as follows: HNHM = Hungarian Natural History Museum, Budapest; IGPP = Museum of the Institute of Genetics, Physiology and Plant Protection, Chişinau; MEIZ = Museum of Entomology, the Institute of Zoology, Chişinau; MSUM = Museum of State University of Moldova, Chişinau; NMEN = National Museum of Ethnography and Natural History, Chişinau.

\section{LIST OF CERAMBYCIDAE FROM THE FOUR MUSEUMS OF CHIŞINĂU}

Nomenclature and systematics follow Althoff \& Danilevsky (1997), DANilevsky $(2014,2019)$ and the Fauna Europaea (https//fauna-eu.org/). The genera and the species are listed in alphabetical order within the subfamilies. For identification, keys of Plavilstshikov $(1958,1965)$ were used.

As for the different combinations, only the most widely used ones mentioned in the cited references are included in the subsequent list.

${ }^{*}=$ species new to the Republic of Moldova. 


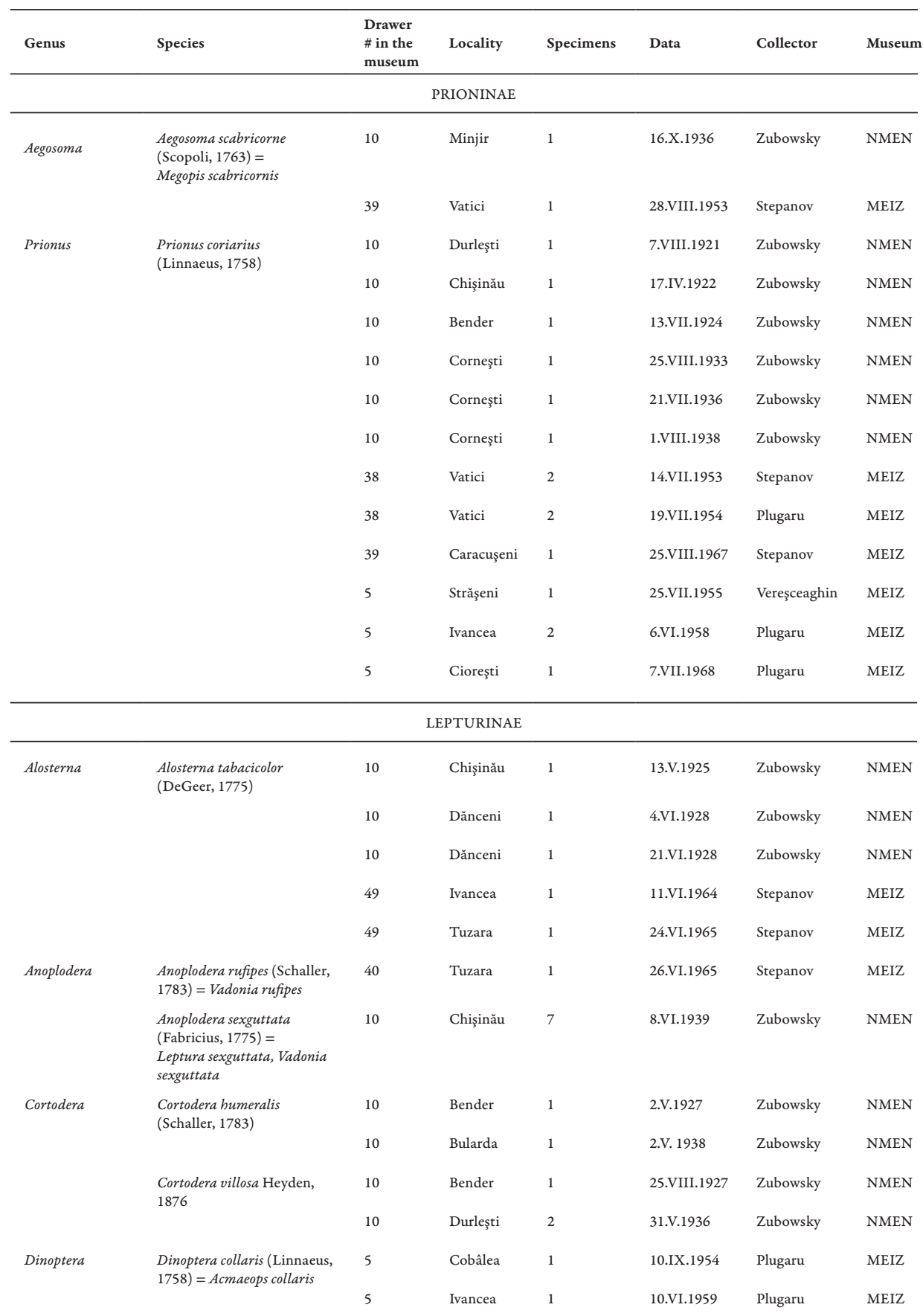




\begin{tabular}{|c|c|c|c|c|c|c|c|}
\hline Genus & Species & $\begin{array}{l}\text { Drawer } \\
\text { \# in the } \\
\text { museum }\end{array}$ & Locality & Specimens & Data & Collector & Museum \\
\hline & $\begin{array}{l}\text { Dinoptera collaris (Linnaeus, } \\
1758)=\text { Acmaeops collaris }\end{array}$ & 5 & Bahmut & 1 & 24.VI.1960 & Plugaru & MEIZ \\
\hline & & 10 & Chişinău & 1 & 20.V.1925 & Zubowsky & NMEN \\
\hline & & 10 & Durleşti & 1 & 28.V.1925 & Zubowsky & NMEN \\
\hline & & 10 & Bender & 2 & 24.V.1925 & Zubowsky & NMEN \\
\hline & & 10 & Bender & 3 & 7.VI.1926 & Zubowsky & NMEN \\
\hline & & 10 & Lozova & 1 & 29.V.1927 & Zubowsky & NMEN \\
\hline & & 49 & Vatici & 1 & 12.VI.1954 & Stepanov & MEIZ \\
\hline & & 49 & Ivancea & 1 & 28.V.1978 & Stepanov & MEIZ \\
\hline Grammoptera & $\begin{array}{l}\text { Grammoptera ruficornis } \\
\text { (Fabricius, 1781) }\end{array}$ & 10 & Bender & 1 & 9.V.1927 & Zubowsky & NMEN \\
\hline \multirow[t]{13}{*}{ Leptura } & $\begin{array}{l}\text { Leptura aethiops Poda, } 1761 \\
=\text { Strangalia aethiops }\end{array}$ & 5 & Bahmut & 2 & 22.VI. 1960 & Ostaficiuc & MEIZ \\
\hline & & 5 & Bahmut & 1 & 25.VI. 1960 & Ostaficiuc & MEIZ \\
\hline & & 5 & Orhei & 1 & 12.VI.1965 & Plugaru & MEIZ \\
\hline & & - & Bahmut & 2 & - & Casianova & MUSM \\
\hline & & - & Criuleni & 6 & 3.VII.1960 & Iacorsan & MUSM \\
\hline & & 10 & Chişinău & 1 & 5.VI.1924 & Zubowsky & NMEN \\
\hline & & 10 & Bender & 2 & 24.V.1925 & Zubowsky & NMEN \\
\hline & & 10 & Bender & 1 & 7.VI.1926 & Zubowsky & NMEN \\
\hline & $\begin{array}{l}\text { Leptura aurulenta (Fabricius, } \\
1792 \text { ) = Strangalia aurulenta }\end{array}$ & 41 & Vatici & 1 & 15.V.1993 & Stepanov & MEIZ \\
\hline & $\begin{array}{l}\text { Leptura quadrifasciata } \\
\text { Linnaeus, } 1758= \\
\text { Strangalia 4-fasciata }\end{array}$ & 49 & Vatici & 1 & 10.V.1953 & Stepanov & MEIZ \\
\hline & & 10 & Chişinău & 1 & 5.VI.1924 & Zubowsky & NMEN \\
\hline & & 10 & Bender & 2 & 24.V.1925 & Zubowsky & NMEN \\
\hline & & 10 & Bender & 1 & 7.VI.1926 & Zubowsky & NMEN \\
\hline \multirow[t]{8}{*}{ Pachytodes } & $\begin{array}{l}\text { Pachytodes cerambyciformis } \\
\text { (Schrank, 1781) }\end{array}$ & 10 & Corneşti & 1 & 8.VI.1973 & Zubowsky & NMEN \\
\hline & $\begin{array}{l}\text { Pachytodes erraticus } \\
\text { (Dalman, 1817) = } \\
\text { Judolia erratica }\end{array}$ & 5 & Ivancea & 4 & 30.VI.1958 & Plugaru & MEIZ \\
\hline & & 5 & Ivancea & 1 & 28.VI.1959 & Plugaru & MEIZ \\
\hline & & 5 & Bahmut & 1 & 27.VI.1960 & Plugaru & MEIZ \\
\hline & & 5 & Calfa & 1 & 6.VII.1978 & Plugaru & MEIZ \\
\hline & & 10 & Goian & 1 & 21.V.1922 & Zubowsky & NMEN \\
\hline & & 10 & Goian & 1 & 11.VI.1922 & Zubowsky & NMEN \\
\hline & & 10 & Goian & 1 & $\begin{array}{l}\text { 20.IV- } \\
\text { 3.VII.1924 }\end{array}$ & Zubowsky & NMEN \\
\hline
\end{tabular}




\begin{tabular}{|c|c|c|c|c|c|c|c|}
\hline Genus & Species & $\begin{array}{l}\text { Drawer } \\
\text { \# in the } \\
\text { museum }\end{array}$ & Locality & Specimens & Data & Collector & Museum \\
\hline & $\begin{array}{l}\text { Pachytodes erraticus } \\
\text { (Dalman, 1817) = } \\
\text { Judolia erratica }\end{array}$ & 10 & Suruceni & 2 & 28.V.1923 & Zubowsky & NMEN \\
\hline & & 10 & Bender & 1 & 7.VI.1926 & Zubowsky & NMEN \\
\hline & & 10 & Chişinău & 1 & 10.VI.1927 & Zubowsky & NMEN \\
\hline & & 40 & Vatici & 1 & 30.V.1953 & Stepanov & MEIZ \\
\hline \multirow[t]{6}{*}{ Pedostrangalia } & $\begin{array}{l}\text { Pedostrangalia revestita } \\
\text { (Linnaeus, } 1767)= \\
\text { Strangalia revestita }\end{array}$ & 11 & Chişinău & 1 & 17.V.1925 & Zubowsky & NMEN \\
\hline & & 11 & Chişinău & 1 & 12.V.1930 & Zubowsky & NMEN \\
\hline & & 11 & Chişinău & 1 & 22.V.1930 & Zubowsky & NMEN \\
\hline & & 11 & Chişinău & 1 & 13.V.1939 & Zubowsky & NMEN \\
\hline & & 11 & Durleşti & 1 & 1.VI.1930 & Zubowsky & NMEN \\
\hline & & 11 & Durleşti & 1 & 23.VI.1933 & Zubowsky & NMEN \\
\hline \multirow[t]{20}{*}{ Pseudovadonia } & $\begin{array}{l}\text { Pseudovadonia livida } \\
\text { (Fabricius, 1776) }= \\
\text { Leptura livida }\end{array}$ & 5 & Ivancea & 1 & 3.VII.1958 & Plugaru & MEIZ \\
\hline & & 5 & Ivancea & 1 & 10.VII.1958 & Plugaru & MEIZ \\
\hline & & 5 & Ivancea & 1 & 16.VII.1958 & Plugaru & MEIZ \\
\hline & & 5 & Ivancea & 1 & 27.V.1959 & Plugaru & MEIZ \\
\hline & & 5 & Ivancea & 1 & 17.VI.59 & Plugaru & MEIZ \\
\hline & & 5 & Ivancea & 1 & 23.VI.1959 & Plugaru & MEIZ \\
\hline & & 5 & Ivancea & 12 & 20.VII.1959 & Plugaru & MEIZ \\
\hline & & 5 & Ivancea & 1 & $6 . X .1959$ & Plugaru & MEIZ \\
\hline & & 5 & Ivancea & 1 & 25.VI. 1960 & Plugaru & MEIZ \\
\hline & & 5 & Calfa & 10 & 6.VII.1978 & Plugaru & MEIZ \\
\hline & & 5 & Cobâlea & 7 & 9.X.1954 & Plugaru & MEIZ \\
\hline & & 5 & Trebisăuți & 1 & 10.VI.1958 & Plugaru & MEIZ \\
\hline & & 5 & Bahmut & 1 & 22.VI.1960 & Plugaru & MEIZ \\
\hline & & 10 & Goian & 1 & 3.VI.1922 & Zubowsky & NMEN \\
\hline & & 10 & Chişinău & 1 & 30.V.1924 & Zubowsky & NMEN \\
\hline & & 10 & Chişinău & 1 & 30.V.1925 & Zubowsky & NMEN \\
\hline & & 10 & Chişinău & 1 & 15.VI.1926 & Zubowsky & NMEN \\
\hline & & 10 & Durleşti & 1 & 28.VI.1928 & Zubowsky & NMEN \\
\hline & & 10 & Bender & 2 & 7-8.VI.1926 & Zubowsky & NMEN \\
\hline & & 10 & Dănceni & 4 & $\begin{array}{l}4-28 . \\
\text { VI.1928 }\end{array}$ & Zubowsky & NMEN \\
\hline
\end{tabular}




\begin{tabular}{|c|c|c|c|c|c|c|c|}
\hline Genus & Species & $\begin{array}{l}\text { Drawer } \\
\text { \# in the } \\
\text { museum }\end{array}$ & Locality & Specimens & Data & Collector & Museum \\
\hline & $\begin{array}{l}\text { Pseudovadonia livida } \\
\text { (Fabricius, 1776) }= \\
\text { Leptura livida }\end{array}$ & 49 & Vatici & 1 & 10.VII.1955 & Stepanov & MEIZ \\
\hline & & 49 & Ivancea & 1 & -.VI.1965 & Stepanov & MEIZ \\
\hline \multirow[t]{21}{*}{ Rhagium } & $\begin{array}{l}{ }^{*} \text { Rhagium inquisitor } \\
\text { (Linnaeus, 1758) }\end{array}$ & 39 & Ivancea & 3 & 10.V.1964 & Stepanov & MEIZ \\
\hline & $\begin{array}{l}\text { Rhagium mordax } \\
\text { (DeGeer, 1775) }\end{array}$ & - & Bahmut & 2 & 20.VI.1956 & - & MUSM \\
\hline & & 10 & Chişinău & 1 & 5.V.1927 & Zubowsky & NMEN \\
\hline & & 10 & Corneşti & 1 & 18.VI.1933 & Zubowsky & NMEN \\
\hline & $\begin{array}{l}\text { Rhagium sycophanta } \\
\text { (Schrank, 1781) }\end{array}$ & 10 & Suruceni & 1 & $\begin{array}{l}28 . V- \\
10 . V I .1927\end{array}$ & Zubowsky & NMEN \\
\hline & & 10 & Leordoaia & 1 & 8.VI.1928 & Zubowsky & NMEN \\
\hline & & 10 & Bularda & 1 & 14.V.1929 & Zubowsky & NMEN \\
\hline & & 10 & Durleşti & 1 & 8.V.1932 & Zubowsky & NMEN \\
\hline & & 11 & Zloți & 1 & 29.V.1986 & Maţiuc & IGPP \\
\hline & & 11 & Lozova & 1 & 16.051986 & Mațiuc & IGPP \\
\hline & & 38 & Ivancea & 2 & 10.V.1963 & Stepanov & MEIZ \\
\hline & & 38 & Vatici & 2 & 12.V.1953 & Stepanov & MEIZ \\
\hline & & 38 & Vatici & 1 & 15.V.1954 & Stepanov & MEIZ \\
\hline & & 39 & Trebujeni & 1 & 26.IV.1959 & Stepanov & MEIZ \\
\hline & & 39 & Ivancea & 5 & 10.V.1964 & Stepanov & MEIZ \\
\hline & & 5 & Ivancea & 1 & 9.V.1958 & Plugaru & MEIZ \\
\hline & & 5 & Ivancea & 1 & 21.V.1958 & Plugaru & MEIZ \\
\hline & & 5 & Ivancea & 1 & 29.V.1959 & Plugaru & MEIZ \\
\hline & & 5 & Ivancea & 1 & 17.VI.1959 & Plugaru & MEIZ \\
\hline & & 5 & Logăneşti & 1 & 14.V.1969 & Plugaru & MEIZ \\
\hline & & 5 & Trebujeni & 1 & 26.IV.1959 & Plugaru & MEIZ \\
\hline \multirow[t]{3}{*}{ Rhamnusium } & $\begin{array}{l}\text { Rhamnusium bicolor } \\
\text { (Schrank, 1781) }\end{array}$ & 10 & Chişinău & 2 & $\begin{array}{l}21-30 . \\
\text { V.1926 }\end{array}$ & Zubowsky & NMEN \\
\hline & & 10 & Durleşti & 1 & 15.V.1924 & Zubowsky & NMEN \\
\hline & & 10 & Durleşti & 1 & 25.V.1929 & Zubowsky & NMEN \\
\hline \multirow[t]{5}{*}{ Rutpela } & $\begin{array}{l}\text { Rutpela maculata (Poda, } \\
1761)=\text { Leptura maculata, }\end{array}$ & - & Bahmut & 3 & 18.VI.1952 & Sereda & MUSM \\
\hline & Strangalia maculata & 10 & Chişinău & 3 & 28.V.1925 & Zubowsky & NMEN \\
\hline & & 10 & Durleşti & 1 & 16.VI.1929 & Zubowsky & NMEN \\
\hline & & 10 & Durleşti & 1 & 1.VI. 1930 & Zubowsky & NMEN \\
\hline & & 10 & Dănceni & 4 & 21.VI.1928 & Zubowsky & NMEN \\
\hline
\end{tabular}




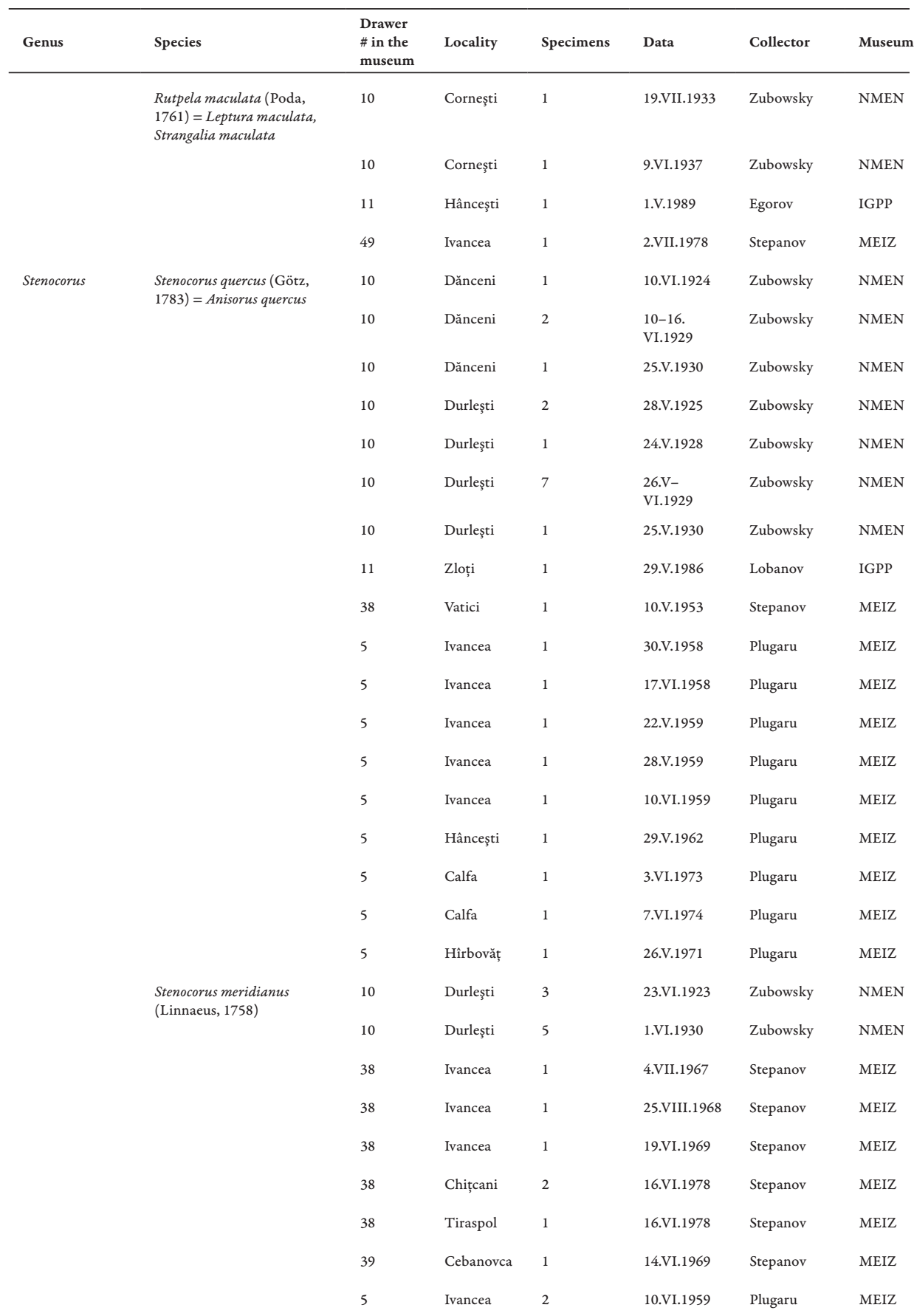




\begin{tabular}{|c|c|c|c|c|c|c|c|}
\hline Genus & Species & $\begin{array}{l}\text { Drawer } \\
\text { \# in the } \\
\text { museum }\end{array}$ & Locality & Specimens & Data & Collector & Museum \\
\hline & $\begin{array}{l}\text { Stenocorus meridianus } \\
\text { (Linnaeus, 1758) }\end{array}$ & 5 & Ivancea & 1 & 13.VI.1960 & Plugaru & MEIZ \\
\hline & & 5 & $\begin{array}{l}\text { Dubăsarii } \\
\text { Vechi }\end{array}$ & 1 & 17.V.1966 & Plugaru & MEIZ \\
\hline & & 5 & Calfa & 1 & 29.VI.1976 & Plugaru & MEIZ \\
\hline \multirow[t]{26}{*}{ Stenurella } & $\begin{array}{l}\text { Stenurella bifasciata } \\
\text { (O. F. Müller, 1776) = }\end{array}$ & 10 & Suruceni & 1 & 28.V.1923 & Zubowsky & NMEN \\
\hline & Strangalia bifasciata & 10 & Călăraşi & 1 & $\begin{array}{l}\text { 20.VII- } \\
\text { 2.VIII.1923 }\end{array}$ & Zubowsky & NMEN \\
\hline & & 10 & Bender & 1 & 14.VI.1924 & Zubowsky & NMEN \\
\hline & & 10 & Durleşti & 1 & 5.VII. 1925 & Zubowsky & NMEN \\
\hline & & 10 & Durleşti & 1 & 28.VI.1928 & Zubowsky & NMEN \\
\hline & & 10 & Chişinău & 2 & 6-7.VI.1926 & Zubowsky & NMEN \\
\hline & & 10 & Chişinău & 1 & 17.VI.1928 & Zubowsky & NMEN \\
\hline & & 10 & Dănceni & 2 & $\begin{array}{l}20-21 . \\
\text { VI.1928 }\end{array}$ & Zubowsky & NMEN \\
\hline & & 49 & Ivancea & 1 & 10.VI.1955 & Stepanov & MEIZ \\
\hline & & 49 & Ivancea & 1 & 15.VI.1955 & Stepanov & MEIZ \\
\hline & $\begin{array}{l}\text { Stenurella melanura } \\
(\text { Linnaeus, } 1758)= \\
\text { Strangalia melanura }\end{array}$ & 11 & Dănceni & 1 & 10.VI.1923 & Zubowsky & NMEN \\
\hline & & 11 & Durleşti & 1 & 5.VI.1926 & Zubowsky & NMEN \\
\hline & & 11 & Durleşti & 1 & 24.VI.1928 & Zubowsky & NMEN \\
\hline & & 40 & Vatici & 1 & 10.VI.1953 & Stepanov & MEIZ \\
\hline & & 40 & Ivancea & 1 & 31.V.1962 & Stepanov & MEIZ \\
\hline & & 40 & Ivancea & 1 & 31.V.1963 & Stepanov & MEIZ \\
\hline & & 49 & Ivancea & 1 & 1.VI.1964 & Stepanov & MEIZ \\
\hline & $\begin{array}{l}\text { Stenurella nigra (Linnaeus, } \\
1758)=\text { Strangalia nigra }\end{array}$ & 11 & Suruceni & 2 & $\begin{array}{l}12-28 . \\
\text { V.1923 }\end{array}$ & Zubowsky & NMEN \\
\hline & & 11 & Chişinău & 1 & 28.V.1923 & Zubowsky & NMEN \\
\hline & & 11 & Chişinău & 1 & 28.V.1925 & Zubowsky & NMEN \\
\hline & & 11 & Durleşti & 2 & 28.VI.1928 & Zubowsky & NMEN \\
\hline & & 11 & Dănceni & 1 & 21.VI.1928 & Zubowsky & NMEN \\
\hline & & 40 & Vatici & 1 & 12.VI.1954 & Stepanov & MEIZ \\
\hline & & 40 & Ivancea & 1 & 30.V.1965 & Stepanov & MEIZ \\
\hline & & 49 & Vatici & 1 & 30.V.1954 & Stepanov & MEIZ \\
\hline & $\begin{array}{l}\text { Stenurella septempunctata } \\
\text { (Fabricius, 1793) = } \\
\text { Strangalia septempunctata, } \\
\text { Leptura septempunctata }\end{array}$ & 11 & Dănceni & 4 & $\begin{array}{l}21-24 . \\
\text { VI.1928 }\end{array}$ & Zubowsky & NMEN \\
\hline
\end{tabular}




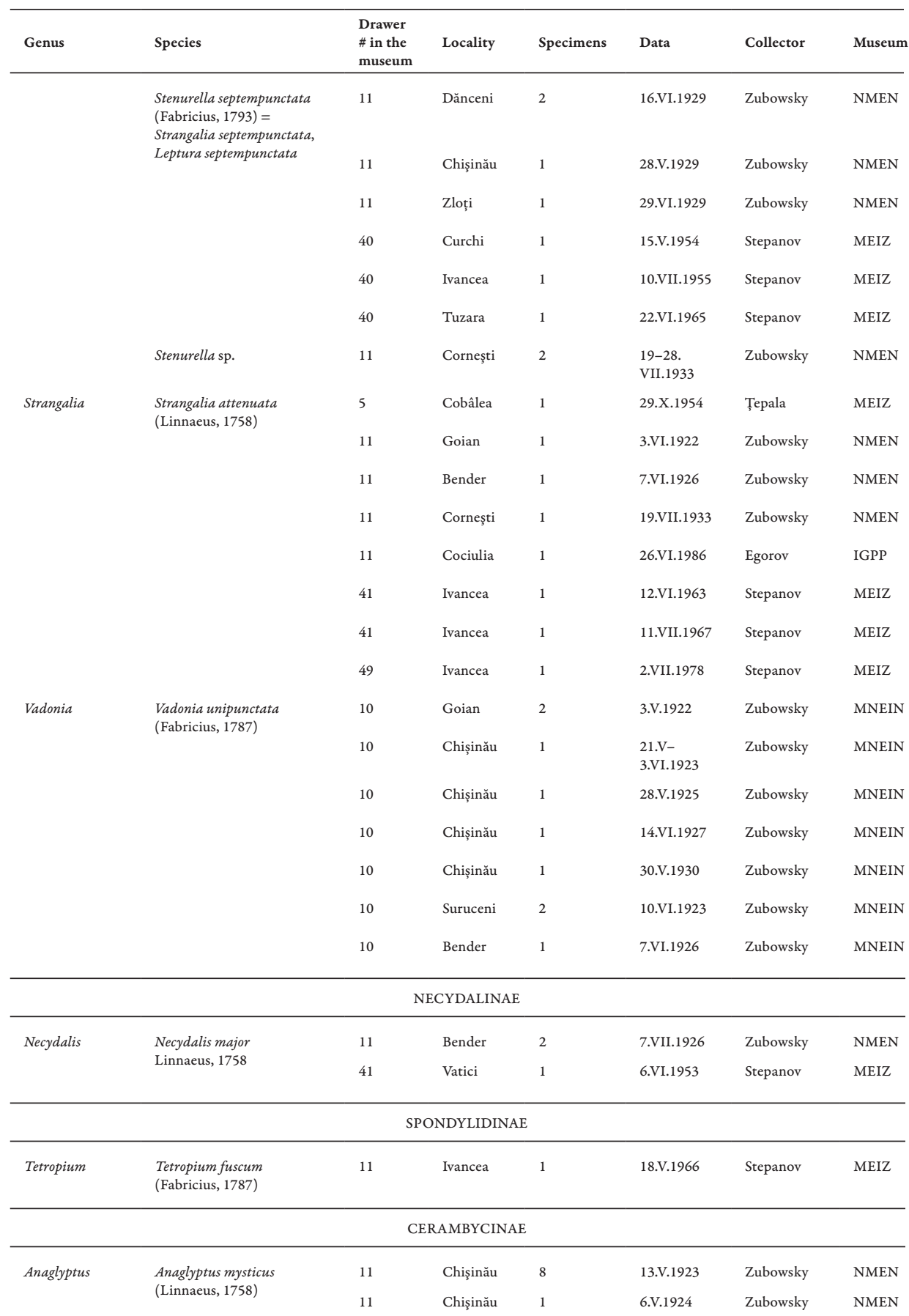




\begin{tabular}{|c|c|c|c|c|c|c|c|}
\hline Genus & Species & $\begin{array}{l}\text { Drawer } \\
\text { \# in the } \\
\text { museum }\end{array}$ & Locality & Specimens & Data & Collector & Museum \\
\hline & $\begin{array}{l}\text { Anaglyptus mysticus } \\
\text { (Linnaeus, 1758) }\end{array}$ & 11 & Chişinău & 1 & 20.V.1925 & Zubowsky & NMEN \\
\hline & & 11 & Durleşti & 1 & 14.V.1922 & Zubowsky & NMEN \\
\hline & & 11 & Durleşti & 1 & 17. V.1931 & Zubowsky & NMEN \\
\hline & & 11 & Suruceni & 1 & 10.VI.1923 & Zubowsky & NMEN \\
\hline & & 11 & Suruceni & 1 & 21.VI.1928 & Zubowsky & NMEN \\
\hline & & 11 & Suruceni & 1 & 28.VI.1928 & Zubowsky & NMEN \\
\hline & & 42 & Vatici & 1 & 10.VI.1953 & Stepanov & MEIZ \\
\hline \multirow[t]{10}{*}{ Aromia } & $\begin{array}{l}\text { Aromia moschata } \\
\text { (Linnaeus, 1758) }\end{array}$ & - & Palanca & 28 & 25.IV.2004 & - & MUSM \\
\hline & & - & Chițcani & 32 & -.VII.1960 & - & MUSM \\
\hline & & 10 & Bender & 5 & 3.VI.1925 & Zubowsky & NMEN \\
\hline & & 43 & Ivancea & 1 & -.VI.1963 & Stepanov & MEIZ \\
\hline & & 43 & Ivancea & 1 & 17.VII.1965 & Stepanov & MEIZ \\
\hline & & 43 & Ivancea & 1 & 20.VI.1967 & Stepanov & MEIZ \\
\hline & & 43 & Onițcani & 1 & -.VI.1964 & Stepanov & MEIZ \\
\hline & & 48 & Vatici & 1 & 17.VI.1954 & Stepanov & MEIZ \\
\hline & & 48 & Ivancea & 1 & 7.VI.1955 & Stepanov & MEIZ \\
\hline & & 5 & Olăneşti & 2 & 7.VI.1961 & Plugaru & MEIZ \\
\hline \multirow[t]{2}{*}{ Axinopalpis } & $\begin{array}{l}\text { Axinopalpis gracilis } \\
\text { (Krynicki, 1832) }\end{array}$ & 11 & Chişinău & 1 & 22.VI.1922 & Zubowsky & NMEN \\
\hline & & 11 & Chişinău & 2 & $\begin{array}{l}15-23 . \\
\text { VI. } 1930\end{array}$ & Zubowsky & NMEN \\
\hline \multirow[t]{4}{*}{ Callimus } & $\begin{array}{l}\text { Callimus angulatus } \\
\text { (Schrank, 1789) }\end{array}$ & 11 & Chişinău & 2 & 7.V.1925 & Zubowsky & NMEN \\
\hline & & 11 & Bularda & 1 & 18.V.1929 & Zubowsky & NMEN \\
\hline & & 11 & Bularda & 1 & 2.V.1938 & Zubowsky & NMEN \\
\hline & & 11 & Străşeni & 1 & 7.IX.1937 & Zubowsky & NMEN \\
\hline \multirow[t]{6}{*}{ Callidium } & $\begin{array}{l}\text { Callidium violaceum } \\
\text { (Linnaeus, 1758) }\end{array}$ & 11 & Bender & 1 & 14.V.1924 & Zubowsky & NMEN \\
\hline & & 11 & Chişinău & 1 & 13.VI.1933 & Zubowsky & NMEN \\
\hline & & 11 & Chişinău & 1 & 12.V.1936 & Zubowsky & NMEN \\
\hline & & 11 & Chişinău & 2 & 20.V.1976 & Crijanovski & IGPP \\
\hline & & 43 & Curchi & 1 & 20.VI.1954 & Stepanov & MEIZ \\
\hline & & 43 & Ivancea & 1 & -.VI.1963 & Stepanov & MEIZ \\
\hline \multirow[t]{3}{*}{ Cerambyx } & $\begin{array}{l}\text { Cerambyx cerdo } \\
\text { (Linnaeus, } 1758 \text { ) }\end{array}$ & - & Bahmut & 19 & 17.VI.1953 & Davidson & MUSM \\
\hline & & - & $\begin{array}{l}\text { Ştefan } \\
\text { Vodă }\end{array}$ & 3 & 10.VII.1952 & Davidson & MUSM \\
\hline & & 11 & Suruceni & 1 & 28.IX.1926 & Zubowsky & NMEN \\
\hline
\end{tabular}




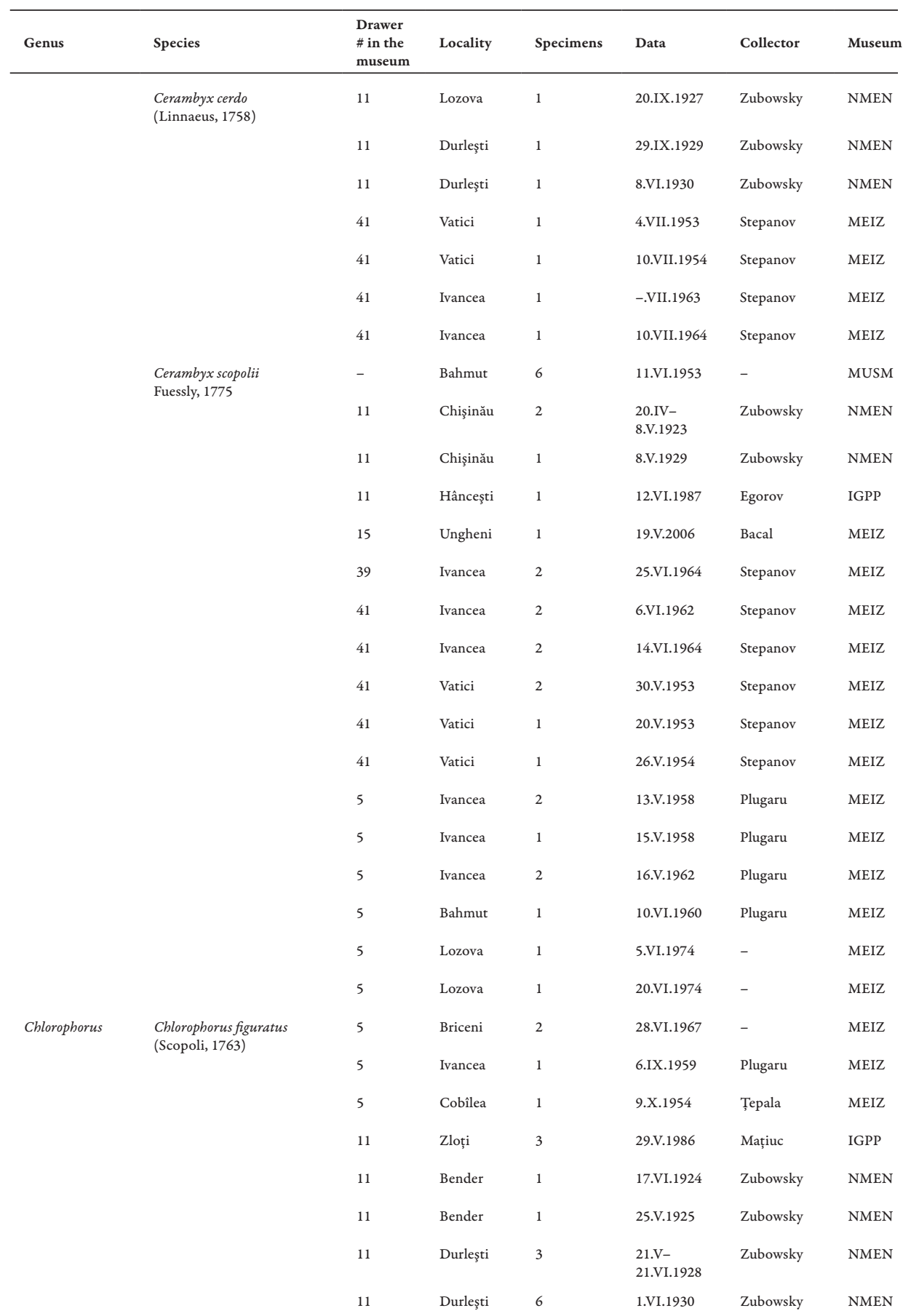




\begin{tabular}{|c|c|c|c|c|c|c|c|}
\hline Genus & Species & $\begin{array}{l}\text { Drawer } \\
\text { \# in the } \\
\text { museum }\end{array}$ & Locality & Specimens & Data & Collector & Museum \\
\hline & $\begin{array}{l}\text { Chlorophorus figuratus } \\
\text { (Scopoli, 1763) }\end{array}$ & 48 & Tuzara & 2 & 20.VI.1965 & Stepanov & MEIZ \\
\hline & $\begin{array}{l}\text { Chlorophorus herbsti } \\
\text { (Brahm, 1790) }\end{array}$ & 11 & Bularda & 1 & 11.VI.1927 & Zubowsky & NMEN \\
\hline & & 11 & Chişinău & 5 & $\begin{array}{l}30 . V-1 . \\
\text { VI.1930 }\end{array}$ & Zubowsky & NMEN \\
\hline & & 43 & Ivancea & 1 & 17.VII.1973 & Stepanov & MEIZ \\
\hline & & 48 & Ivancea & 1 & 16.VI.1964 & Stepanov & MEIZ \\
\hline & $\begin{array}{l}\text { Chlorophorus sartor } \\
\text { (O. F. Müller, 1766) }\end{array}$ & 5 & Ivancea & 1 & 7.VI.1958 & Plugaru & MEIZ \\
\hline & & 5 & Ivancea & 1 & 7.VII.1958 & Plugaru & MEIZ \\
\hline & & 5 & Ivancea & 1 & 3.VII.1959 & Plugaru & MEIZ \\
\hline & & 5 & Ivancea & 16 & 18.VII.1959 & Plugaru & MEIZ \\
\hline & & 5 & Ivancea & 17 & 20.VII.1959 & Plugaru & MEIZ \\
\hline & & 5 & Ivancea & 12 & 21.VII.1959 & Plugaru & MEIZ \\
\hline & & 5 & Ivancea & 1 & 25.VII.1958 & Plugaru & MEIZ \\
\hline & & 11 & Bender & 2 & 14.VI.1924 & Zubowsky & NMEN \\
\hline & & 11 & Chişinău & 2 & $\begin{array}{l}\text { 10.V- } \\
22 . V I .1924\end{array}$ & Zubowsky & NMEN \\
\hline & & 11 & Chişinău & 2 & 13.VII.1926 & Zubowsky & NMEN \\
\hline & & 11 & Durleşti & 1 & 4.VII.1925 & Zubowsky & NMEN \\
\hline & & 15 & Cociulia & 2 & 19.VII.2004 & Bacal & MEIZ \\
\hline & & 15 & Codru & 2 & 6.VII.2019 & Bacal & MEIZ \\
\hline & & 42 & Vatici & 1 & 10.VI.1953 & Stepanov & MEIZ \\
\hline & & 49 & Ivancea & 1 & 19.VI.1963 & Stepanov & MEIZ \\
\hline & $\begin{array}{l}\text { Chlorophorus varius } \\
\text { (O. F. Müller, 1766) }\end{array}$ & 11 & Bender & 1 & 17.VI.1924 & Zubowsky & NMEN \\
\hline & & 11 & Chişinău & 1 & 13.VII.1926 & Zubowsky & NMEN \\
\hline & & 11 & Durleşti & 1 & 4.VII.1931 & Zubowsky & NMEN \\
\hline & & 11 & Palanca & 1 & 24.VI.1982 & Tuganov & IGPP \\
\hline & & 11 & Palanca & 1 & 6.VII.1982 & Tuganov & IGPP \\
\hline & & 11 & Palanca & 3 & 29.VI.1987 & Tuganov & IGPP \\
\hline & & 15 & Codru & 4 & 6.VII.2019 & Bacal & MEIZ \\
\hline & & 43 & Vatici & 1 & 20.V.1953 & Stepanov & MEIZ \\
\hline & & 43 & Ivancea & 2 & 23.VII.1970 & Stepanov & MEIZ \\
\hline & Chlorophorus sp. & 11 & Chişinău & 1 & 9.VI.1927 & Zubowsky & NMEN \\
\hline Clytus & Clytus rhamni Germar, 1817 & 5 & Chişinău & 1 & 19.VI.1966 & - & MEIZ \\
\hline & & 11 & Zloți & 1 & 29.V.1986 & Mațiuc & IGPP \\
\hline
\end{tabular}




\begin{tabular}{|c|c|c|c|c|c|c|c|}
\hline Genus & Species & $\begin{array}{l}\text { Drawer } \\
\text { \# in the } \\
\text { museum }\end{array}$ & Locality & Specimens & Data & Collector & Museum \\
\hline & Clytus rhamni Germar, 1817 & 11 & Bender & 3 & $\begin{array}{l}14 . V I-17 . \\
\text { VI.1924 }\end{array}$ & Zubowsky & NMEN \\
\hline & & 11 & Chişinău & 1 & 10.VI.1928 & Zubowsky & NMEN \\
\hline \multirow[t]{8}{*}{ Hylotrupes } & $\begin{array}{l}\text { Hylotrupes bajulus } \\
\text { (Linnaeus, 1758) }\end{array}$ & 11 & Chişinău & 1 & 17.IV.1922 & Zubowsky & NMEN \\
\hline & & 11 & Chişinău & 1 & 14.VII.1923 & Zubowsky & NMEN \\
\hline & & 11 & Chişinău & 1 & 25.VI.1924 & Zubowsky & NMEN \\
\hline & & 11 & Chişinău & 2 & 5.VIII. 1928 & Zubowsky & NMEN \\
\hline & & 11 & Chişinău & 1 & 18.VII.1929 & Zubowsky & NMEN \\
\hline & & 11 & Bender & 1 & 4.VII.1923 & Zubowsky & NMEN \\
\hline & & 43 & Vatici & 1 & 25.VII.1953 & Stepanov & MEIZ \\
\hline & & 43 & Vatici & 1 & 10.VIII.1953 & Stepanov & MEIZ \\
\hline \multirow[t]{8}{*}{ Isotomus } & $\begin{array}{l}\text { Isotomus speciosus } \\
\text { (Schneider, 1787) }\end{array}$ & 11 & Chişinău & 1 & 14.V.1924 & Zubowsky & NMEN \\
\hline & & 11 & Corneşti & 1 & 24.VII.1933 & Zubowsky & NMEN \\
\hline & & 11 & Corneşti & 1 & 22.VIII.1933 & Zubowsky & NMEN \\
\hline & & 11 & Corneşti & 1 & 12.VI.1936 & Zubowsky & NMEN \\
\hline & & 11 & Corneşti & 1 & 2.VII.1936 & Zubowsky & NMEN \\
\hline & & 42 & Vatici & 1 & 06.VII.1953 & Stepanov & MEIZ \\
\hline & & 42 & Vatici & 1 & 10.VII.1953 & Stepanov & MEIZ \\
\hline & & 46 & Ivancea & 1 & 26.VII.1978 & Stepanov & MEIZ \\
\hline \multirow[t]{2}{*}{ Leioderes } & $\begin{array}{l}\text { Leioderes kollari } \\
\text { Redtenbacher, } 1849\end{array}$ & 11 & Chişinău & 6 & $\begin{array}{l}30 . \mathrm{V}- \\
3 . \mathrm{VI} .1933\end{array}$ & Zubowsky & NMEN \\
\hline & & 11 & Durleşti & 1 & 14.V.1939 & Zubowsky & NMEN \\
\hline \multirow[t]{5}{*}{ Obrium } & $\begin{array}{l}\text { Obrium brunneum } \\
\text { (Fabricius, 1792) }\end{array}$ & 43 & Ivancea & 2 & 5.VI.1962 & Stepanov & MEIZ \\
\hline & $\begin{array}{l}\text { Obrium cantharinum } \\
\text { (Linnaeus, 1767) }\end{array}$ & 11 & Bularda & 2 & 7.VI.1928 & Zubowsky & NMEN \\
\hline & & 43 & Ivancea & 1 & 7.IV.1966 & Stepanov & MEIZ \\
\hline & & 43 & Ivancea & 1 & 5.VI.1982 & Stepanov & MEIZ \\
\hline & & 49 & Ivancea & 1 & 17.VI.1966 & Stepanov & MEIZ \\
\hline \multirow[t]{3}{*}{ Palaeocallidium } & $\begin{array}{l}\text { Palaeocallidium coriaceum } \\
\text { (Paykull, 1800) }= \\
\text { Callidium coriaceum }\end{array}$ & 11 & Zloți & 1 & 29.V.1986 & Egorov & IGPP \\
\hline & & 11 & Chişinău & 1 & 20.V.1976 & Crijanovski & IGPP \\
\hline & & 43 & Ivancea & 1 & -.V.1966 & Stepanov & MEIZ \\
\hline Paraphymatodes & $\begin{array}{l}\text { Paraphymatodes fasciatus } \\
\text { (Villers, 1789) }\end{array}$ & 43 & Ivancea & 1 & 8.V.1968 & Stepanov & MEIZ \\
\hline Phymatodes & $\begin{array}{l}\text { Phymatodes puncticollis } \\
\text { (Mulsant, 1862) = } \\
\text { Phymatoderus puncticollis, } \\
\text { Reitteroderus puncticollis }\end{array}$ & 11 & Dănceni & 1 & $25 . V .1930$ & Zubowsky & NMEN \\
\hline
\end{tabular}




\begin{tabular}{|c|c|c|c|c|c|c|c|}
\hline Genus & Species & $\begin{array}{l}\text { Drawer } \\
\text { \# in the } \\
\text { museum }\end{array}$ & Locality & Specimens & Data & Collector & Museum \\
\hline & $\begin{array}{l}\text { Phymatodes rufipes } \\
\text { (Fabricius, 1776) }= \\
\text { Phymatodellus rufipes }\end{array}$ & 11 & Chişinău & 1 & 14.VI.1922 & Zubowsky & NMEN \\
\hline & $\begin{array}{l}\text { Phymatodes testaceus ab. } \\
\text { praeustus (Linnaeus, 1758) = } \\
\text { Callidium testaceus }\end{array}$ & 11 & Chişinău & 1 & 16.VI.1920 & Zubowsky & NMEN \\
\hline & & 11 & Chişinău & 1 & 19.IV.1923 & Zubowsky & NMEN \\
\hline & & 11 & Chişinău & 1 & 13.VI.1933 & Zubowsky & NMEN \\
\hline & & 43 & Ivancea & 1 & 14.VII.1964 & Stepanov & MEIZ \\
\hline & & 43 & Vatici & 1 & 30.V.1953 & Stepanov & MEIZ \\
\hline & $\begin{array}{l}\text { Phymatodes testaceus ab. } \\
\text { variabilis (Linnaeus, 1758) }\end{array}$ & 11 & Chişinău & 3 & $\begin{array}{l}25-27 \\
\text { V.1923 }\end{array}$ & Zubowsky & NMEN \\
\hline & & 11 & Chişinău & 1 & 13.V.1925 & Zubowsky & NMEN \\
\hline & & 11 & Chişinău & 1 & 27.V.1929 & Zubowsky & NMEN \\
\hline & & 11 & Chişinău & 1 & 16.VI.1929 & Zubowsky & NMEN \\
\hline \multirow[t]{2}{*}{ Poecilium } & $\begin{array}{l}\text { Poecilium alni } \\
\text { (Linnaeus, 1767) }= \\
\text { Phymatodes alni }\end{array}$ & 11 & Chişinău & 1 & 13.V.1922 & Zubowsky & NMEN \\
\hline & & 11 & Chişinău & 2 & $9-12 . V .1924$ & Zubowsky & NMEN \\
\hline \multirow[t]{12}{*}{ Plagionotus } & $\begin{array}{l}\text { Plagionotus arcuatus } \\
\text { (Linnaeus, 1758) }\end{array}$ & 11 & Chişinău & 2 & $\begin{array}{l}\text { 30.V- } \\
\text { 13.VI.1922 }\end{array}$ & Zubowsky & NMEN \\
\hline & & 11 & Chişinău & 1 & 6.VII.1924 & Zubowsky & NMEN \\
\hline & & 11 & Chişinău & 1 & 3.V.1928 & Zubowsky & NMEN \\
\hline & & 11 & Bender & 1 & 14.V.1924 & Zubowsky & NMEN \\
\hline & & 43 & Vatici & 1 & 17.VI.1953 & Stepanov & MEIZ \\
\hline & & 43 & Vatici & 1 & 20.V.1954 & Stepanov & MEIZ \\
\hline & & 43 & Ivancea & 1 & -.VI.1965 & Stepanov & MEIZ \\
\hline & & 48 & Ivancea & 2 & 18.V.1963 & Stepanov & MEIZ \\
\hline & & 48 & Vatici & 1 & 20.V.1963 & Stepanov & MEIZ \\
\hline & $\begin{array}{l}\text { Plagionotus detritus } \\
\text { Linnaeus, } 1758\end{array}$ & 48 & Ivancea & 1 & 30.V.1964 & Stepanov & MEIZ \\
\hline & & 48 & Ivancea & 1 & 26.VI.1974 & Stepanov & MEIZ \\
\hline & $\begin{array}{l}\text { Plagionotus floralis (Pallas, } \\
1776 \text { ) = Echinocerus floralis }\end{array}$ & 11 & Dănceni & 3 & $\begin{array}{l}21-28 . \\
\text { VI. } 1928\end{array}$ & Zubowsky & NMEN \\
\hline \multirow[t]{5}{*}{ Purpuricenus } & $\begin{array}{l}\text { Purpuricenus budensis } \\
\text { (Götz, 1783) }\end{array}$ & 11 & Chişinău & 1 & 20.VI.1924 & Zubowsky & NMEN \\
\hline & $\begin{array}{l}\text { Purpuricenus kaehleri } \\
\text { Linnaeus, } 1758\end{array}$ & - & Bahmut & 3 & 28.VI.1955 & Lîsenko & MUSM \\
\hline & & - & Bahmut & 1 & 15.VII.1960 & Petrica & MUSM \\
\hline & & 11 & Chişinău & 1 & 7.VI.1927 & Zubowsky & NMEN \\
\hline & & 11 & Chişinău & 1 & 5.VII. 1930 & Zubowsky & NMEN \\
\hline
\end{tabular}




\begin{tabular}{|c|c|c|c|c|c|c|c|}
\hline Genus & Species & $\begin{array}{l}\text { Drawer } \\
\text { \# in the } \\
\text { museum }\end{array}$ & Locality & Specimens & Data & Collector & Museum \\
\hline & $\begin{array}{l}\text { Purpuricenus kaebleri } \\
\text { Linnaeus, } 1758\end{array}$ & 11 & Chişinău & 1 & 22.VI.1936 & Zubowsky & NMEN \\
\hline & & 42 & Vatici & 1 & -.V.1953 & Stepanov & MEIZ \\
\hline & & 42 & Vatici & 1 & 20.V.1954 & Stepanov & MEIZ \\
\hline & & 46 & Tuzara & 1 & 22.VI.1965 & Stepanov & MEIZ \\
\hline Pyrrbidium & $\begin{array}{l}\text { Pyrrhidium sanguineum } \\
\text { (Linnaeus, 1758) }\end{array}$ & 11 & Chișinău & 3 & $\begin{array}{l}\text { 12.III- } \\
\text { 18.IV.1922 }\end{array}$ & Zubowsky & NMEN \\
\hline \multirow[t]{13}{*}{ Ropalopus } & $\begin{array}{l}\text { Ropalopus clavipes } \\
\text { (Fabricius, 1775) }\end{array}$ & 11 & Bularda & 1 & 27.VI.1928 & Zubowsky & NMEN \\
\hline & & 11 & Dănceni & 1 & 28.VI.1928 & Zubowsky & NMEN \\
\hline & & 11 & Corneşti & 2 & 25.VII.1936 & Zubowsky & NMEN \\
\hline & & 43 & Dubăsari & 1 & -.VII.1953 & Stepanov & MEIZ \\
\hline & & 43 & Ivancea & 1 & 28.VI.1967 & Stepanov & MEIZ \\
\hline & $\begin{array}{l}\text { Ropalopus macropus } \\
\text { (Germar, 1824) }\end{array}$ & 11 & Chişinău & 2 & 12.V.1923 & Zubowsky & NMEN \\
\hline & & 11 & Chişinău & 1 & 20.V.1924 & Zubowsky & NMEN \\
\hline & & 11 & Chişinău & 2 & 22.V.1926 & Zubowsky & NMEN \\
\hline & & 11 & Chişinău & 1 & 13.VI.1933 & Zubowsky & NMEN \\
\hline & & 11 & Durleşti & 1 & 1.VI.1930 & Zubowsky & NMEN \\
\hline & & 43 & Ivancea & 1 & 20.VII.1973 & Stepanov & MEIZ \\
\hline & & 49 & Ivancea & 1 & 10.V.1955 & Stepanov & MEIZ \\
\hline & & 49 & Ivancea & 1 & 14.VII.1963 & Stepanov & MEIZ \\
\hline \multirow[t]{2}{*}{ Rosalia } & $\begin{array}{l}\text { Rosalia alpina } \\
\text { (Linnaeus, 1758) }\end{array}$ & 43 & Vatici & 1 & 15.VII.1953 & Stepanov & MEIZ \\
\hline & & 43 & Ivancea & 1 & 8.VII.1963 & Stepanov & MEIZ \\
\hline \multirow[t]{6}{*}{ Stenopterus } & $\begin{array}{l}\text { Stenopterus rufus } \\
\text { (Linnaeus, 1767) }\end{array}$ & 11 & Chişinău & 1 & 2.VI.1924 & Zubowsky & NMEN \\
\hline & & 11 & Chişinău & 1 & 12.VI.1928 & Zubowsky & NMEN \\
\hline & & 11 & Bender & 2 & 7.VI.1926 & Zubowsky & NMEN \\
\hline & & 11 & Bender & 1 & 23.V.1929 & Zubowsky & NMEN \\
\hline & & 11 & Dănceni & 1 & 28.VI.1928 & Zubowsky & NMEN \\
\hline & & 11 & Corneşti & 1 & 6.VI.1937 & Zubowsky & NMEN \\
\hline \multirow[t]{6}{*}{ Xylotrechus } & $\begin{array}{l}\text { Xylotrechus antilope } \\
\text { (Schönherr, 1817) }\end{array}$ & 11 & Chişinău & 1 & 29.VI.1931 & Zubowsky & NMEN \\
\hline & & 43 & Ivancea & 1 & 28.V.1964 & Stepanov & MEIZ \\
\hline & & 43 & Ivancea & 1 & 8.VI.1964 & Stepanov & MEIZ \\
\hline & $\begin{array}{l}\text { Xylotrechus arvicola } \\
\text { (Olivier, 1795) }\end{array}$ & 11 & $\begin{array}{l}\text { Vadul-lui- } \\
\text { Vodă }\end{array}$ & 1 & 11.VI.1920 & Zubowsky & NMEN \\
\hline & $\begin{array}{l}\text { Xylotrechus rusticus } \\
\text { (Linnaeus, 1758) }\end{array}$ & 11 & Bender & 1 & 7.VI.1926 & Zubowsky & NMEN \\
\hline & & 11 & Bender & 6 & 6.V.1927 & Zubowsky & NMEN \\
\hline
\end{tabular}




\begin{tabular}{|c|c|c|c|c|c|c|c|}
\hline Genus & Species & $\begin{array}{l}\text { Drawer } \\
\text { \# in the } \\
\text { museum }\end{array}$ & Locality & Specimens & Data & Collector & Museum \\
\hline \multicolumn{8}{|c|}{ LAMIINAE } \\
\hline \multirow[t]{28}{*}{ Agapanthia } & $\begin{array}{l}\text { Agapanthia asphodeli } \\
\text { Latreille, } 1804\end{array}$ & 45 & Ivancea & 1 & -.VI.1965 & Stepanov & MEIZ \\
\hline & $\begin{array}{l}\text { Agapanthia dabli } \\
\text { (Richter, 1821) }\end{array}$ & 11 & Chişinău & 1 & 15.VII.1912 & Zubowsky & NMEN \\
\hline & & 11 & Bender & 3 & 24.V.1925 & Zubowsky & NMEN \\
\hline & & 11 & Hruşova & 1 & 10.VI.1928 & Zubowsky & NMEN \\
\hline & & 11 & Corneşti & 1 & 19.VII.1933 & Zubowsky & NMEN \\
\hline & & 48 & Tiraspol & 1 & -.VII.1968 & Stepanov & MEIZ \\
\hline & & 48 & Onițcahi & 1 & -.VI.1965 & Stepanov & MEIZ \\
\hline & $\begin{array}{l}\text { *Agapanthia lederi } \\
\text { Ganglbauer, } 1884= \\
\text { Agapanthia helianthi } \\
\text { Plavilshtshikov, } 1935\end{array}$ & 11 & Zloți & 1 & 29.V.1986 & Mațiuc & IGPP \\
\hline & $\begin{array}{l}\text { Agapanthia villosoviridescens } \\
\text { (DeGeer, 1775) }\end{array}$ & 5 & Lozova & 1 & 6.VI.1974 & - & MEIZ \\
\hline & & 11 & Bender & 5 & 24.V.1925 & Zubowsky & NMEN \\
\hline & & 11 & Bender & 1 & 14.VII.1929 & Zubowsky & NMEN \\
\hline & & 11 & Bularda & 1 & 19.V.1927 & Zubowsky & NMEN \\
\hline & & 11 & Corneşti & 2 & 9.VI.1937 & Zubowsky & NMEN \\
\hline & & 45 & Vatici & 1 & 10.VIII.1953 & Stepanov & MEIZ \\
\hline & & 48 & Ivancea & 1 & -.VII.1964 & Stepanov & MEIZ \\
\hline & & 48 & Ivancea & 1 & 3.VI.1976 & Stepanov & MEIZ \\
\hline & $\begin{array}{l}\text { *Agapanthia } \\
\text { villosoviridescens var. } \\
\text { subchalybaea } \text { Reitter, } 1898= \\
\text { Agapanthia subchalybaea }\end{array}$ & 45 & Ivancea & 1 & 17.VI.1969 & Stepanov & MEIZ \\
\hline & $\begin{array}{l}\text { Agapanthia violacea } \\
\text { (Fabricius, 1775) }\end{array}$ & 11 & Chişinău & 1 & $\begin{array}{l}11 . V- \\
29 . V I .1922\end{array}$ & Zubowsky & NMEN \\
\hline & & 11 & Chişinău & 3 & $\begin{array}{l}\text { 30.IV- } \\
\text { 30.VI.1923 }\end{array}$ & Zubowsky & NMEN \\
\hline & & 11 & Chişinău & 1 & 19.V.1924 & Zubowsky & NMEN \\
\hline & & 11 & Chişinău & 1 & 1.V.1925 & Zubowsky & NMEN \\
\hline & & 11 & Chişinău & 1 & 17.V.1925 & Zubowsky & NMEN \\
\hline & & 11 & Chişinău & 1 & 22.V.1925 & Zubowsky & NMEN \\
\hline & & 11 & Goian & 1 & $\begin{array}{l}\text { 3.IV- } \\
\text { 21.V.1922 }\end{array}$ & Zubowsky & NMEN \\
\hline & & 11 & Goian & 2 & 20.VI.1924 & Zubowsky & NMEN \\
\hline & & 11 & Dănceni & 1 & $\begin{array}{l}\text { 24.IV- } \\
\text { 8.V.1922 }\end{array}$ & Zubowsky & NMEN \\
\hline & & 11 & Dănceni & 1 & 16.V.1931 & Zubowsky & NMEN \\
\hline & & 45 & Ivancea & 1 & 3.VI.1963 & Stepanov & MEIZ \\
\hline
\end{tabular}




\begin{tabular}{|c|c|c|c|c|c|c|c|}
\hline Genus & Species & $\begin{array}{l}\text { Drawer } \\
\text { \# in the } \\
\text { museum }\end{array}$ & Locality & Specimens & Data & Collector & Museum \\
\hline & $\begin{array}{l}\text { Agapanthia violacea } \\
\text { (Fabricius, 1775) }\end{array}$ & 48 & Tudora & 2 & 26.VI.1965 & Stepanov & MEIZ \\
\hline \multirow[t]{2}{*}{ Agapanthiola } & $\begin{array}{l}\text { Agapanthiola leucaspis } \\
\text { (Steven, 1817) }\end{array}$ & 11 & Dănceni & 1 & 10.VI.1929 & Zubowsky & NMEN \\
\hline & & 11 & Dănceni & 2 & 16.V.1937 & Zubowsky & NMEN \\
\hline \multirow[t]{6}{*}{ Anaesthetis } & $\begin{array}{l}\text { Anaesthetis testacea } \\
\text { (Fabricius, 1781) }\end{array}$ & 11 & Chişinău & 1 & $\begin{array}{l}\text { 21.V- } \\
\text { 3.VI.1923 }\end{array}$ & Zubowsky & NMEN \\
\hline & & 11 & Chişinău & 1 & 30.V.1929 & Zubowsky & NMEN \\
\hline & & 11 & Durlesti & 1 & 25.VI.1924 & Zubowsky & NMEN \\
\hline & & 11 & Suruceni & 1 & 10.VI.1923 & Zubowsky & NMEN \\
\hline & & 11 & Dănceni & 1 & 21.VI.1928 & Zubowsky & NMEN \\
\hline & & 11 & Dănceni & 1 & 28.VI.1928 & Zubowsky & NMEN \\
\hline Calamobius & $\begin{array}{l}\text { Calamobius filum } \\
\text { (Rossi, 1790) }\end{array}$ & 11 & Chişinău & 3 & 8.VI.1937 & Zubowsky & NMEN \\
\hline \multirow[t]{20}{*}{ Dorcadion } & $\begin{array}{l}\text { Dorcadion equestre } \\
(\text { Laxmann, 1770) = } \\
\text { Pedestredorcadion equestre }\end{array}$ & 11 & Chişinău & 1 & 18.V.1923 & Zubowsky & NMEN \\
\hline & & 11 & Chişinău & 1 & 11.V.1924 & Zubowsky & NMEN \\
\hline & & 11 & Chişinău & 1 & 20.IV.1930 & Zubowsky & NMEN \\
\hline & & 11 & Sculeni & 1 & 20.IV.1925 & Zubowsky & NMEN \\
\hline & & 46 & Tiraspol & 1 & -.IV.1964 & Stepanov & MEIZ \\
\hline & $\begin{array}{l}\text { Dorcadion fulvum } \\
\text { (Scopoli, 1763) = } \\
\text { Carinatodorcadion fulvum }\end{array}$ & - & Bahmut & 3 & 19.VI.1951 & Litvinskaia & MUSM \\
\hline & & - & Bahmut & 3 & 13.VI.1953 & - & MUSM \\
\hline & & 11 & Chişinău & 1 & 16.V.1923 & Zubowsky & NMEN \\
\hline & & 11 & Chişinău & 2 & 8.V.1924 & Zubowsky & NMEN \\
\hline & & 11 & Suruceni & 2 & $\begin{array}{l}\text { 15.V- } \\
20 . V .1923\end{array}$ & Zubowsky & NMEN \\
\hline & & 11 & Sculeni & 1 & 20.IV.1925 & Zubowsky & NMEN \\
\hline & & 42 & Vatici & 1 & 5.IV.1953 & Stepanov & MEIZ \\
\hline & & 42 & Vatici & 1 & 25.V.1953 & Stepanov & MEIZ \\
\hline & & 46 & Ivancea & 1 & 20.V.1976 & Stepanov & MEIZ \\
\hline & & 46 & Ivancea & 1 & 30.V.1976 & Stepanov & MEIZ \\
\hline & & 5 & Trebujeni & 4 & 25.IV.1959 & Plugaru & MEIZ \\
\hline & & 5 & Trebujeni & 3 & 27.IV.1959 & Plugaru & MEIZ \\
\hline & & 5 & Vatici & 4 & 20.V.1958 & Plugaru & MEIZ \\
\hline & & 5 & Bahmut & 3 & 1.VII.1963 & Plugaru & MEIZ \\
\hline & & 5 & Bahmut & 4 & 30.VII. 1960 & Plugaru & MEIZ \\
\hline
\end{tabular}




\begin{tabular}{|c|c|c|c|c|c|c|c|}
\hline Genus & Species & $\begin{array}{l}\text { Drawer } \\
\text { \# in the } \\
\text { museum }\end{array}$ & Locality & Specimens & Data & Collector & Museum \\
\hline & $\begin{array}{l}\text { Dorcadion fulvum } \\
\text { (Scopoli, 1763) = } \\
\text { Carinatodorcadion fulvum }\end{array}$ & 5 & Bahmut & 1 & 23.VII.1961 & Plugaru & MEIZ \\
\hline & & 5 & Ivancea & 1 & 14.V.1958 & Plugaru & MEIZ \\
\hline & & 5 & Ivancea & 1 & 21.V.1958 & Plugaru & MEIZ \\
\hline & & 5 & Leuşeni & 1 & 5.V.1966 & Plugaru & MEIZ \\
\hline & $\begin{array}{l}\text { Dorcadion holosericeum } \\
\text { Krynicky, } 1832\end{array}$ & - & Bahmut & 10 & 6.V.1955 & Belova & MUSM \\
\hline & & 42 & Vatici & 1 & 10.VI.1953 & Stepanov & MEIZ \\
\hline & & 42 & Ivancea & 1 & 7.IV.1955 & Stepanov & MEIZ \\
\hline & $\begin{array}{l}\text { Dorcadion pedestre } \\
(\text { Poda, } 1761)= \\
\text { Pedestredorcadion pedestre }\end{array}$ & 11 & Cociulia & 1 & 26.VI.1986 & Lobanov & IGPP \\
\hline & & 11 & Zloți & 1 & 29.V.1986 & Lobanov & IGPP \\
\hline & & 11 & Chişinău & 1 & 18.V.1923 & Zubowsky & NMEN \\
\hline & & 11 & Chişinău & 1 & 10.V.1925 & Zubowsky & NMEN \\
\hline & & 11 & Durleşti & 1 & 20.V.1925 & Zubowsky & NMEN \\
\hline & & 11 & Sculeni & 1 & 20.IV.1925 & Zubowsky & NMEN \\
\hline & & 11 & Corneşti & 1 & 8.VI.1937 & Zubowsky & NMEN \\
\hline & & 15 & Cociulia & 1 & 21.V.2006 & Bacal & MEIZ \\
\hline & & 42 & Ivancea & 2 & 10.V.1964 & Stepanov & MEIZ \\
\hline & & 46 & Ivancea & 2 & -.IV.1965 & Stepanov & MEIZ \\
\hline & & 5 & Trebujeni & 3 & 25.IV.1959 & Vereşceaghin & MEIZ \\
\hline & $\begin{array}{l}\text { Dorcadion pusillum } \\
\text { Küster, } 1847= \\
\text { Pedestredorcadion pusillum }\end{array}$ & 11 & Durleşti & 1 & 14.V.1922 & Zubowsky & NMEN \\
\hline & & 11 & Durleşti & 1 & 14.IV.1923 & Zubowsky & NMEN \\
\hline & & 11 & Chişinău & 1 & 11.IV.1923 & Zubowsky & NMEN \\
\hline & & 11 & Chişinău & 4 & $\begin{array}{l}5-20 . \\
\text { IV.1924 }\end{array}$ & Zubowsky & NMEN \\
\hline & & 11 & Dănceni & 1 & 22.V.1932 & Zubowsky & NMEN \\
\hline & $\begin{array}{l}\text { Dorcadion sericatum } \\
\text { (Sahlberg, 1823) }\end{array}$ & 11 & Chişinău & 1 & 8.V.1922 & Zubowsky & NMEN \\
\hline & & 11 & Chişinău & 2 & $\begin{array}{l}15-18 . \\
\text { V.1923 }\end{array}$ & Zubowsky & NMEN \\
\hline & & 11 & Chişinău & 1 & 30.IV.1925 & Zubowsky & NMEN \\
\hline & & 11 & Chişinău & 1 & 10.V.1925 & Zubowsky & NMEN \\
\hline & & 11 & Chişinău & 1 & 28.V.1925 & Zubowsky & NMEN \\
\hline & $\begin{array}{l}\text { Dorcadion caucasicum } \\
\text { Küster, } 1847\end{array}$ & 42 & Vatici & 1 & 15.IV.1953 & Stepanov & MEIZ \\
\hline & & 42 & Vatici & 1 & 20.IV.1953 & Stepanov & MEIZ \\
\hline
\end{tabular}




\begin{tabular}{|c|c|c|c|c|c|c|c|}
\hline \multirow[t]{2}{*}{ Genus } & Species & $\begin{array}{l}\text { Drawer } \\
\# \text { in the } \\
\text { museum }\end{array}$ & Locality & Specimens & Data & Collector & Museum \\
\hline & $\begin{array}{l}\text { Dorcadion caucasicum } \\
\text { Küster, } 1847\end{array}$ & 42 & Ivancea & 1 & 23.IV.1966 & Stepanov & MEIZ \\
\hline & $\begin{array}{l}\text { Dorcadion tauricum } \\
\text { Waltl, } 1838\end{array}$ & 11 & Durleşti & 4 & $\begin{array}{l}\text { 24.IV- } \\
\text { 4.V.1922 }\end{array}$ & Zubowsky & NMEN \\
\hline & & 11 & Durleşti & 1 & 10.IV.1923 & Zubowsky & NMEN \\
\hline & & 11 & Chişinău & 1 & 28.IV.1923 & Zubowsky & NMEN \\
\hline & & 11 & Chişinău & 1 & 16.V.1923 & Zubowsky & NMEN \\
\hline & & 11 & Chişinău & 1 & 1.V.1925 & Zubowsky & NMEN \\
\hline & & 11 & Chişinău & 1 & 11.IV.1933 & Zubowsky & NMEN \\
\hline & & 11 & Bender & 2 & 20.IV.1925 & Zubowsky & NMEN \\
\hline & & 5 & Vatici & 4 & 20.V.1958 & Plugaru & MEIZ \\
\hline & & 5 & Trebujeni & 8 & 26.IV.1959 & Plugaru & MEIZ \\
\hline & & 5 & Trebujeni & 6 & 27.IV.1959 & Plugaru & MEIZ \\
\hline & & 5 & Trebujeni & 1 & 25.IV.1959 & Vereşceaghin & MEIZ \\
\hline & & 5 & Trebujeni & 2 & 27.IV.1959 & Vereşceaghin & MEIZ \\
\hline & & 5 & Ivancea & 3 & 12.V.1958 & Plugaru & MEIZ \\
\hline & & 5 & Ivancea & 1 & 16.V.1958 & Plugaru & MEIZ \\
\hline & & 5 & Ivancea & 1 & 21.V.1958 & Plugaru & MEIZ \\
\hline & & 5 & Ivancea & 2 & 22.V.1958 & Plugaru & MEIZ \\
\hline & & 5 & Ivancea & 1 & 28.V.1958 & Plugaru & MEIZ \\
\hline & & 5 & Ivancea & 1 & 30.V.1958 & Plugaru & MEIZ \\
\hline & & 5 & Ivancea & 1 & 31.V.1958 & Plugaru & MEIZ \\
\hline & & 5 & Ivancea & 1 & 12.VI.1959 & Plugaru & MEIZ \\
\hline & & 5 & Ivancea & 4 & 14.V.1958 & Vereşceaghin & MEIZ \\
\hline & & 5 & Ivancea & 1 & 15.V.1958 & Vereşceaghin & MEIZ \\
\hline & & 5 & Ivancea & 1 & 30.V.1958 & Vereşceaghin & MEIZ \\
\hline & & 5 & Ivancea & 1 & 25.IV.1959 & Vereşceaghin & MEIZ \\
\hline & & 5 & Bahmut & 1 & 5.VII. 1960 & Plugaru & MEIZ \\
\hline & & 5 & Leuşeni & 2 & 5.V.1966 & Vereşceaghin & MEIZ \\
\hline & & 5 & Chişinău & 1 & 10.V.1963 & Plugaru & MEIZ \\
\hline & & 5 & Logăneşti & 1 & 14.V.1969 & Plugaru & MEIZ \\
\hline & Dorcadion decipiens & 5 & Trebujeni & 1 & 26.IV.1959 & Plugaru & MEIZ \\
\hline & Germar, 1824 & & & & & & \\
\hline & & 5 & Trebujeni & 2 & 27.IV.1959 & Vereşceaghin & MEIZ \\
\hline & & 5 & Socoleni & 2 & 9.V.1993 & Ostaficiuc & MEIZ \\
\hline
\end{tabular}




\begin{tabular}{|c|c|c|c|c|c|c|c|}
\hline Genus & Species & $\begin{array}{l}\text { Drawer } \\
\text { \# in the } \\
\text { museum }\end{array}$ & Locality & Specimens & Data & Collector & Museum \\
\hline & Dorcadion sp. & 11 & Dănceni & 3 & 4.VI.1928 & Zubowsky & NMEN \\
\hline \multirow[t]{12}{*}{ Exocentrus } & $\begin{array}{l}\text { Exocentrus adspersus } \\
\text { Mulsant, } 1846\end{array}$ & 11 & Palanca & 1 & 7.VII.1928 & Zubowsky & NMEN \\
\hline & & 44 & Ivancea & 1 & 5.VI.1962 & Stepanov & MEIZ \\
\hline & & 48 & Ivancea & 1 & 20.VI.1967 & Stepanov & MEIZ \\
\hline & $\begin{array}{l}\text { Exocentrus lusitanus } \\
\text { (Linnaeus, 1767) }\end{array}$ & 11 & Chișinău & 1 & 8.VI.1936 & Zubowsky & NMEN \\
\hline & & 11 & Durleşti & 1 & 10.VI.1936 & Zubowsky & NMEN \\
\hline & & 11 & Corneşti & 1 & 15.VII.1938 & Zubowsky & NMEN \\
\hline & & 44 & Ivancea & 1 & 5.VI.1962 & Stepanov & MEIZ \\
\hline & & 44 & Ivancea & 1 & 13.VI.1965 & Stepanov & MEIZ \\
\hline & & 44 & Ivancea & 1 & 31.VII.1978 & Stepanov & MEIZ \\
\hline & $\begin{array}{l}\text { Exocentrus stierlini } \\
\text { Ganglbauer, } 1883\end{array}$ & 11 & Briceni & 1 & 7.IX.1917 & Zubowsky & NMEN \\
\hline & & 11 & Chişinău & 1 & $\begin{array}{l}\text { 21.V- } \\
3 . V I .1923\end{array}$ & Zubowsky & NMEN \\
\hline & Exocentrus sp. & 44 & Ivancea & 1 & 20.VII.1973 & Stepanov & MEIZ \\
\hline \multirow[t]{5}{*}{ Leiopus } & $\begin{array}{l}\text { Leiopus nebulosus } \\
\text { (Linnaeus, 1758) }\end{array}$ & 11 & Chişinău & 1 & 5.VI.1923 & Zubowsky & NMEN \\
\hline & & 11 & Chişinău & 1 & 19.VI.1925 & Zubowsky & NMEN \\
\hline & & 11 & Chişinău & 2 & $\begin{array}{l}15-30 . \\
\text { V.1926 }\end{array}$ & Zubowsky & NMEN \\
\hline & & 11 & Dănceni & 1 & 4.VII.1931 & Zubowsky & NMEN \\
\hline & & 11 & Chişinău & 1 & 19.V.1974 & Crijanovski & IGPP \\
\hline \multirow[t]{12}{*}{ Mesosa } & $\begin{array}{l}\text { Mesosa curculionoides } \\
\text { (Linnaeus, 1761) }\end{array}$ & 11 & Durleşti & 1 & 14.V.1922 & Zubowsky & NMEN \\
\hline & & 11 & Chişinău & 2 & $\begin{array}{l}\text { 25.IV- } \\
9 . V .1923\end{array}$ & Zubowsky & NMEN \\
\hline & & 11 & Chişinău & 1 & 26.V.1925 & Zubowsky & NMEN \\
\hline & & 11 & Chişinău & 2 & 2-4.V.1926 & Zubowsky & NMEN \\
\hline & & 46 & Vatici & 1 & 4.V.1953 & Stepanov & MEIZ \\
\hline & $\begin{array}{l}\text { Mesosa nebulosa } \\
\text { (Fabricius, 1781) = } \\
\text { Aphelocnemia nebulosa }\end{array}$ & 11 & Bender & 2 & 24.V.1925 & Zubowsky & NMEN \\
\hline & & 11 & Chişinău & 1 & 24.V.1925 & Zubowsky & NMEN \\
\hline & & 11 & Chişinău & 1 & 16.VIII. 1925 & Zubowsky & NMEN \\
\hline & & 11 & Chişinău & 1 & 22.V.1926 & Zubowsky & NMEN \\
\hline & & 11 & Chişinău & 1 & 5.VI.1928 & Zubowsky & NMEN \\
\hline & & 11 & Durleşti & 1 & 4.V.1930 & Zubowsky & NMEN \\
\hline & & 44 & Vatici & 1 & 1.V.1953 & Stepanov & MEIZ \\
\hline Morimus & $\begin{array}{l}\text { Morimus funereus } \\
\text { Mulsant, } 1863= \\
\text { Morimus asper funereus }\end{array}$ & - & Cobîlca & 5 & 4.VI.1953 & - & MUSM \\
\hline
\end{tabular}




\begin{tabular}{|c|c|c|c|c|c|c|c|}
\hline Genus & Species & $\begin{array}{l}\text { Drawer } \\
\text { \# in the } \\
\text { museum }\end{array}$ & Locality & Specimens & Data & Collector & Museum \\
\hline & $\begin{array}{l}\text { Morimus funereus } \\
\text { Mulsant, } 1863= \\
\text { Morimus asper funereus }\end{array}$ & - & Ivancea & 12 & 5.VII.2005 & - & MUSM \\
\hline & & 11 & Străşeni & 1 & 11.VI.1924 & Zubowsky & NMEN \\
\hline & & 11 & Chişinău & 1 & 4.V.1926 & Zubowsky & NMEN \\
\hline & & 11 & Palanca & 3 & $\begin{array}{l}12-29 . \\
\text { V.1927 }\end{array}$ & Zubowsky & NMEN \\
\hline & & 11 & Bularda & 1 & 7.V.1938 & Zubowsky & NMEN \\
\hline & & 44 & Ivancea & 2 & 15.V.1955 & Stepanov & MEIZ \\
\hline & & 44 & Ivancea & 3 & -.V.1956 & Stepanov & MEIZ \\
\hline & & 44 & Ivancea & 1 & -.V.1964 & Stepanov & MEIZ \\
\hline & & 46 & Ivancea & 1 & -.V.1955 & Stepanov & MEIZ \\
\hline & & 46 & Ivancea & 2 & 8.V.1964 & Stepanov & MEIZ \\
\hline & & 46 & Ivancea & 1 & 10.V.1964 & Stepanov & MEIZ \\
\hline & & 5 & Ivancea & 1 & 31.V.1958 & Plugaru & MEIZ \\
\hline & & 5 & Ivancea & 1 & 15.VII.1958 & Plugaru & MEIZ \\
\hline & & 5 & Ivancea & 2 & 29.VII.1958 & Plugaru & MEIZ \\
\hline & & 5 & Ivancea & 1 & 25.V.1959 & Plugaru & MEIZ \\
\hline & & 5 & Bahmut & 2 & 8.VI.1960 & Plugaru & MEIZ \\
\hline & & 5 & Logăneşti & 1 & 14.V.1969 & Plugaru & MEIZ \\
\hline & & 5 & Ivancea & 1 & 14.IV.1958 & Vereşceaghin & MEIZ \\
\hline & & 5 & Orhei & 3 & 11.IV.1966 & - & MEIZ \\
\hline \multirow[t]{2}{*}{ Musaria } & $\begin{array}{l}\text { Musaria affinis } \\
\text { (Harrer, 1784) }\end{array}$ & 12 & Corneşti & 3 & 14.VI.1937 & Zubowsky & NMEN \\
\hline & & 12 & Corneşti & 5 & $\begin{array}{l}\text { 26.VI- } \\
\text { 2.VII.1938 }\end{array}$ & Zubowsky & NMEN \\
\hline \multirow[t]{10}{*}{ Neodorcadion } & $\begin{array}{l}\text { Neodorcadion bilineatum } \\
\text { (Germar, 1824) }\end{array}$ & 11 & Chişinău & 5 & 7-17.V.1923 & Zubowsky & NMEN \\
\hline & & 11 & Chişinău & 1 & 7.V.1925 & Zubowsky & NMEN \\
\hline & & 11 & Bender & 3 & 24.VI.1925 & Zubowsky & NMEN \\
\hline & & 11 & Dişcova & 2 & 12.VII.1939 & Zubowsky & NMEN \\
\hline & & 44 & Vatici & 2 & -.V.1953 & Stepanov & MEIZ \\
\hline & & 44 & Vatici & 1 & 5.V.1954 & Stepanov & MEIZ \\
\hline & & 46 & Tiraspol & 1 & 18.VI.1978 & Stepanov & MEIZ \\
\hline & & 46 & Ivancea & 1 & 3.V.1976 & Stepanov & MEIZ \\
\hline & & 5 & Vatici & 2 & 20.V.1958 & Plugaru & MEIZ \\
\hline & & 5 & Vatici & 1 & 26.IV.1959 & Plugaru & MEIZ \\
\hline
\end{tabular}




\begin{tabular}{|c|c|c|c|c|c|c|c|}
\hline Genus & Species & $\begin{array}{l}\text { Drawer } \\
\text { \# in the } \\
\text { museum }\end{array}$ & Locality & Specimens & Data & Collector & Museum \\
\hline \multirow[t]{13}{*}{ Oberea } & $\begin{array}{l}\text { Oberea erythrocephala } \\
\text { (Schrank, 1776) }\end{array}$ & 45 & Chițcani & 1 & 16.VI.1978 & Stepanov & MEIZ \\
\hline & & 12 & Bender & 6 & 24.V.1925 & Zubowsky & NMEN \\
\hline & & 12 & Bender & 1 & 7.VI.1926 & Zubowsky & NMEN \\
\hline & & 11 & Comrat & 1 & 26.VI.1986 & Egorov & IGPP \\
\hline & $\begin{array}{l}\text { Oberea euphorbiae } \\
\text { (Germar, 1813) }\end{array}$ & 12 & Bender & 7 & 24.V.1925 & Zubowsky & NMEN \\
\hline & $\begin{array}{l}\text { Oberea linearis } \\
\text { (Linnaeus, 1761) }\end{array}$ & 12 & Corneşti & 1 & 28.VI.1937 & Zubowsky & NMEN \\
\hline & & 48 & Tiraspol & 3 & 16.VI.1978 & Stepanov & MEIZ \\
\hline & & 48 & Chițcani & 1 & 16.VI.1978 & Stepanov & MEIZ \\
\hline & $\begin{array}{l}\text { *Oberea oculata } \\
(\text { Linnaeus, } 1758)\end{array}$ & 5 & Lozova & 1 & 6.VI.1974 & - & MEIZ \\
\hline & Oberea sp. & 12 & Bender & 1 & 24.V.1925 & Zubowsky & NMEN \\
\hline & & 12 & Bender & 1 & 7.VI.1926 & Zubowsky & NMEN \\
\hline & & 12 & Bender & 2 & 8.VI.1927 & Zubowsky & NMEN \\
\hline & & 12 & Bender & 1 & 10.V.1930 & Zubowsky & NMEN \\
\hline \multirow[t]{18}{*}{ Phytoecia } & $\begin{array}{l}\text { Phytoecia caerulea } \\
\text { (Scopoli, 1772) }\end{array}$ & 12 & Chişinău & 1 & $\begin{array}{l}\text { 22.V- } \\
\text { 3.VI.1923 }\end{array}$ & Zubowsky & NMEN \\
\hline & & 12 & Chişinău & 2 & 22.V.1925 & Zubowsky & NMEN \\
\hline & & 12 & Bender & 1 & 2.V.1926 & Zubowsky & NMEN \\
\hline & & 12 & Bender & 1 & 6.V.1927 & Zubowsky & NMEN \\
\hline & $\begin{array}{l}\text { Phytoecia caerulescens } \\
(\text { Scopoli, 1763) }=\end{array}$ & 12 & Bender & 1 & 7.VI.1926 & Zubowsky & NMEN \\
\hline & Opsilia coerulescens & 12 & Chişinău & 1 & 10.VI.1928 & Zubowsky & NMEN \\
\hline & & 12 & Lozova & 1 & 29.V.1927 & Zubowsky & NMEN \\
\hline & & 12 & Corneşti & 1 & 19.VII. 1933 & Zubowsky & NMEN \\
\hline & & 12 & Corneşti & 1 & 16.VI.1937 & Zubowsky & NMEN \\
\hline & & 12 & Suruceni & 7 & $\begin{array}{l}\text { 28.IV- } \\
\text { 15.V.1923 }\end{array}$ & Zubowsky & NMEN \\
\hline & & 12 & Chişinău & 4 & 22.V.1925 & Zubowsky & NMEN \\
\hline & & 12 & Durleşti & 1 & 7.VI.1929 & Zubowsky & NMEN \\
\hline & & 45 & Vatici & 1 & 24.V.1953 & Stepanov & MEIZ \\
\hline & & 48 & Vatici & 1 & 25.V.1953 & Stepanov & MEIZ \\
\hline & $\begin{array}{l}\text { Phytoecia cylindrica } \\
\text { (Linnaeus, 1758) }\end{array}$ & 5 & Chişinău & 2 & 25.V.1925 & - & MEIZ \\
\hline & & 12 & Durleşti & 1 & 3.V.1936 & Zubowsky & NMEN \\
\hline & & 12 & Durleşti & 1 & 12.V.1936 & Zubowsky & NMEN \\
\hline & & 12 & Cornești & 3 & 14.VI.1937 & Zubowsky & NMEN \\
\hline
\end{tabular}




\begin{tabular}{|c|c|c|c|c|c|c|c|}
\hline Genus & Species & $\begin{array}{l}\text { Drawer } \\
\# \text { in the } \\
\text { museum }\end{array}$ & Locality & Specimens & Data & Collector & Museum \\
\hline & $\begin{array}{l}\text { Phytoecia cylindrica } \\
\text { (Linnaeus, 1758) }\end{array}$ & 45 & Vatici & 1 & 25.V.1953 & Stepanov & MEIZ \\
\hline & $\begin{array}{l}\text { Phytoecia icterica } \\
\text { (Schaller, 1783) }\end{array}$ & 12 & Chişinău & 1 & 18.VI.1922 & Zubowsky & NMEN \\
\hline & & 12 & Chişinău & 1 & $\begin{array}{l}\text { 23.IV- } \\
\text { 6.V.1923 }\end{array}$ & Zubowsky & NMEN \\
\hline & & 12 & Chişinău & 4 & $\begin{array}{l}10 . V-5 . \\
\text { VI.1924 }\end{array}$ & Zubowsky & NMEN \\
\hline & & 12 & Chişinău & 3 & $\begin{array}{l}29.1 V- \\
5 . V .1925\end{array}$ & Zubowsky & NMEN \\
\hline & & 12 & Chişinău & 1 & 4.V.1926 & Zubowsky & NMEN \\
\hline & & 12 & Chişinău & 1 & 2.VI.1929 & Zubowsky & NMEN \\
\hline & & 12 & Chişinău & 1 & 29.V.1930 & Zubowsky & NMEN \\
\hline & & 12 & Durleşti & 1 & 10.V.1925 & Zubowsky & NMEN \\
\hline & & 12 & Durleşti & 1 & 14.V.1929 & Zubowsky & NMEN \\
\hline & & 12 & Durleşti & 1 & $9 . V .1939$ & Zubowsky & NMEN \\
\hline & & 12 & Goian & 1 & 11.V.1924 & Zubowsky & NMEN \\
\hline & & 12 & Goian & 1 & 2.V.1940 & Zubowsky & NMEN \\
\hline & $\begin{array}{l}\text { Phytoecia nigricornis } \\
\text { (Fabricius, 1781) }\end{array}$ & 12 & Chişinău & 1 & 18.V.1922 & Zubowsky & NMEN \\
\hline & & 12 & Chişinău & 2 & $\begin{array}{l}23 . \mathrm{V}- \\
10 . \mathrm{VI} .1923\end{array}$ & Zubowsky & NMEN \\
\hline & & 12 & Chişinău & 3 & $\begin{array}{l}20-22 . \\
\text { V.1925 }\end{array}$ & Zubowsky & NMEN \\
\hline & & 12 & Chişinău & 1 & 10.VI.1928 & Zubowsky & NMEN \\
\hline & & 12 & Chişinău & 1 & 30.V.1926 & Zubowsky & NMEN \\
\hline & & 12 & Bender & 2 & 7.VI.1926 & Zubowsky & NMEN \\
\hline & & 45 & Vatici & 1 & 25.V.1963 & Stepanov & MEIZ \\
\hline & & 45 & Vatici & 1 & 10.VI.1963 & Stepanov & MEIZ \\
\hline & $\begin{array}{l}\text { Phytoecia pustulata } \\
\text { (Schrank, 1776) }\end{array}$ & 12 & Goian & 1 & 4.VI.1922 & Zubowsky & NMEN \\
\hline & & 12 & Chişinău & 3 & $\begin{array}{l}\text { 27.IV- } \\
\text { 13.V.1923 }\end{array}$ & Zubowsky & NMEN \\
\hline & & 12 & Chişinău & 1 & 2.VII.1938 & Zubowsky & NMEN \\
\hline & & 12 & Suruceni & 1 & 15.V.1923 & Zubowsky & NMEN \\
\hline & $\begin{array}{l}\text { Phytoecia virgula } \\
\text { (Charpentier, 1825) }\end{array}$ & 12 & Chişinău & 1 & 5.V.1925 & Zubowsky & NMEN \\
\hline & & 12 & Bender & 1 & 6.V.1925 & Zubowsky & NMEN \\
\hline & & 12 & Bender & 1 & 7.VI.1926 & Zubowsky & NMEN \\
\hline & & 12 & Dănceni & 2 & 4.VI.1928 & Zubowsky & NMEN \\
\hline & & 12 & Dănceni & 2 & $\begin{array}{l}\text { 20.V- } \\
\text { 14.VI.1929 }\end{array}$ & Zubowsky & NMEN \\
\hline
\end{tabular}




\begin{tabular}{|c|c|c|c|c|c|c|c|}
\hline Genus & Species & $\begin{array}{l}\text { Drawer } \\
\text { \# in the } \\
\text { museum }\end{array}$ & Locality & Specimens & Data & Collector & Museum \\
\hline & $\begin{array}{l}\text { Phytoecia virgula } \\
\text { (Charpentier, 1825) }\end{array}$ & 45 & Ivancea & 1 & 21.V.1976 & Stepanov & MEIZ \\
\hline & Phytoecia sp. & 12 & Dănceni & 1 & 15.V.1938 & Zubowsky & NMEN \\
\hline \multirow[t]{9}{*}{ Pilemia } & $\begin{array}{l}\text { Pilemia hirsutula } \\
\text { (Frölich, 1793) }\end{array}$ & 12 & Bender & 2 & 7.VI.1926 & Zubowsky & NMEN \\
\hline & & 12 & Chişinău & 1 & 16.VI.1933 & Zubowsky & NMEN \\
\hline & & 12 & Corneşti & 1 & 20.VII.1939 & Zubowsky & NMEN \\
\hline & & 48 & Onițcani & 2 & -.VI.1965 & Stepanov & MEIZ \\
\hline & $\begin{array}{l}\text { Pilemia tigrina } \\
\text { (Mulsant, 1851) }\end{array}$ & 12 & Chişinău & 1 & 11.V.1922 & Zubowsky & NMEN \\
\hline & & 12 & Chişinău & 2 & $8-21 . V .1923$ & Zubowsky & NMEN \\
\hline & & 12 & Chişinău & 1 & 24.V.1926 & Zubowsky & NMEN \\
\hline & & 12 & Durleşti & 1 & 25.V.1924 & Zubowsky & NMEN \\
\hline & & 12 & Bender & 1 & 7.VI.1926 & Zubowsky & NMEN \\
\hline \multirow[t]{4}{*}{ Pogonocherus } & $\begin{array}{l}\text { Pogonocherus hispidus } \\
\text { (Linnaeus, 1758) }\end{array}$ & 5 & Chişinău & 1 & 25.V.1977 & Ostaficiuc & MEIZ \\
\hline & & 11 & Durleşti & 1 & 26.V.1929 & Zubowsky & NMEN \\
\hline & $\begin{array}{l}\text { *Pogonocherus hispidulus } \\
\text { (Piller et Mitterpacher, } \\
1783 \text { ) }\end{array}$ & 44 & Ivancea & 1 & 5.VI.1955 & Stepanov & MEIZ \\
\hline & & 44 & Ivancea & 1 & 15.V.1978 & Stepanov & MEIZ \\
\hline \multirow[t]{12}{*}{ Saperda } & $\begin{array}{l}\text { Saperda punctata } \\
\text { (Linnaeus, 1767) }\end{array}$ & 11 & Chişinău & 1 & 9.VI.1925 & Zubowsky & NMEN \\
\hline & & 45 & Vatici & 1 & 10.VI.1954 & Stepanov & MEIZ \\
\hline & & 45 & Ivancea & 1 & 13.VI.1964 & Stepanov & MEIZ \\
\hline & & 48 & Vatici & 1 & 10.VI.1954 & Stepanov & MEIZ \\
\hline & $\begin{array}{l}\text { Saperda populnea } \\
\text { (Linnaeus, 1758) }= \\
\text { Compsidia populnea }\end{array}$ & 11 & Hagimus & 1 & 7.V.1927 & Zubowsky & NMEN \\
\hline & & 11 & Bularda & 1 & 18.V.1929 & Zubowsky & NMEN \\
\hline & & 11 & Bularda & 5 & 14.V.1930 & Zubowsky & NMEN \\
\hline & & 11 & Străşeni & 1 & 7.V.1937 & Zubowsky & NMEN \\
\hline & $\begin{array}{l}\text { Saperda octopunctata } \\
\text { (Scopoli, 1772) }\end{array}$ & 11 & Chişinău & 1 & 8.VI.1928 & Zubowsky & NMEN \\
\hline & $\begin{array}{l}\text { Saperda scalaris } \\
\text { (Linnaeus, 1758) }\end{array}$ & 11 & Bularda & 1 & 11.V.1928 & Zubowsky & NMEN \\
\hline & & 11 & Bularda & 5 & 11.V.1930 & Zubowsky & NMEN \\
\hline & & 11 & Corneşti & 1 & 18.VII.1933 & Zubowsky & NMEN \\
\hline \multirow[t]{3}{*}{ Tetrops } & $\begin{array}{l}\text { Tetrops praeustus } \\
\text { (Linnaeus, 1758) }\end{array}$ & 12 & Chişinău & 1 & 13.V.1923 & Zubowsky & NMEN \\
\hline & & 12 & Chişinău & 1 & 20.V.1924 & Zubowsky & NMEN \\
\hline & & 12 & Chişinău & 5 & $\begin{array}{l}\text { 29.IV- } \\
7 . V .1925\end{array}$ & Zubowsky & NMEN \\
\hline
\end{tabular}




\begin{tabular}{llllllll}
\hline Genus & Species & $\begin{array}{l}\text { Drawer } \\
\text { \#n the } \\
\text { museum }\end{array}$ & Locality & Specimens & Data & Collector & Museum \\
\hline $\begin{array}{l}\text { Tetropspraeustus } \\
\text { (Linnaeus, 1758) }\end{array}$ & 12 & Chişinău & 1 & 21. V.1936 & Zubowsky & NMEN \\
& 45 & Vatici & 1 & 3. V.1954 & Stepanov & MEIZ \\
& 45 & Ivancea & 1 & 12. V.1955 & Stepanov & MEIZ \\
& 48 & Vatici & 1 & 15. V.1954 & Stepanov & MEIZ \\
\hline
\end{tabular}

\section{RESULTS}

In the four collections of Chişinău, 1153 specimens representing 121 species belonging to 60 genera and 6 subfamilies of Cerambycidae were found. These allowed us to complete the list of the family with 5 species new to the country: Agapanthia lederi, A. subchalybaea, Orbea oculata, Pogonocherus hispidulus and Rhagium inquisitor (marked with asterisk in the table above).

The most speciose subfamily is Lamiinae, which is represented by 457 specimens belonging to 51 species and 17 genera, followed by Cerambycinae with 392 specimens belonging to 38 species and 23 genera, then Lepturinae with 283 specimens belonging to 28 species and 16 genera and Prioninae with 17 specimens belonging to 2 species and 2 genera. Two subfamilies, Necydalinae and Spondylidinae are with 3 and one specimen, respectively, belonging to one species and one genus only.

Analysis per collections - The richest Cerambycidae collection is kept in the National Museum of Ethnography and Natural History, and consists of 554 specimens belonging to 101 species, 55 genera and 6 subfamilies, collected in 20 localities: city of Chişinău, town Bender, village Durleşti, v. Dănceni, t. Corneşti, v. Suriceni, v. Bularda, v. Goian, v. Lozova, v. Sculeni, t. Străşeni, v. Palanca, t. Briceni, t. Călăraşi, v. Dişcova, v. Hagimus, v. Hruşova, v. Minjir, v. Zloți and t. Vadul-lui-Vodă. The majority of the species was collected around c. Chişinău, villages Durleşti and Dănceni, and around towns Bender and Corneşti.

The highest number of specimens is assigned to Phytoecia caerulescens (17), followed by Anaglyptus mysticus (15) and Agapanthia violacea (13). Three species, Grammoptera ruficornis, Xylotrechus arvicola and Saperda octopunctata, are found only in this collection having represented by one specimen each. Cerambycidae species of the National Museum of Ethnography and Natural History are mentioned in the catalogue by DeRJanschi et al. (2016) and the paper of MILLER \& ZUBOWSKY (1917).

The second largest insect collection is kept in the Museum of Entomology, Institute of Zoology, which consists of 437 specimens belonging to 76 species, 42 genera and 6 subfamilies, collected in 28 localities: village Bahmut, v. Calfa, v. Caracuşeni, v. Cebanovca, v. Cioreşti, c. Chişinău, v. Chițcani, v. Curchi, 
t. Dubăsari, v. Dubăsari Vechi, t. Hânceşti, v. Hîrbovăț, v. Ivancea, t. Leuşeni, v. Logăneşti, v. Lozova, v. Olăneşti, t. Orhei, v. Onițcani, v. Palanca, v. Socoleni, t. Străşeni, t. Tiraspol, v. Trebisăuți, v. Trebujeni, v. Tudora, v. Tuzara and v. Vatici. Most of of the specimens deposited in this museum were collected in Ivancea and Vatici. The highest numbers of specimens are assigned to Chlorophorus sartor (61), Dorcadion tauricum (55) and Pseudovadonia livida (52).

The following species are represented by singletons: Leptura aurulenta, Leptura quadrifasciata, Tetropium fuscum, Paraphymatodes fasciatus, Pogonocherus hispidus, Oberea oculata, Agapanthia asphodeli and Agapanthia subchalybaea. These species are not present in the other studied collections.

The Cerambycidae collection stored in the Museum of the State University of Moldova includes 138 specimens belonging to 10 species, 7 genera and 3 subfamilies. Insects were collected in seven localities: v. Bahmut, c. Chişinău, v. Cobâlca, v. Criuleni, v. Ivancea, v. Palanca and t. Ştefan Vodă. The majority of them were collected in Bahmut by students. The most abundant species in this museum is Aromia moschata with 60 specimens, followed by Cerambyx cerdo with 22 specimens.

The collection of Cerambycidae kept in the Museum of the Institute of Genetics, Physiology and Plant Protection includes only 24 Cerambycidae specimens, belonging to 14 species, 13 genera and 3 subfamilies, collected in seven localities: v. Zloți, c. Chişinău, v. Cociulia, t. Hânceşti, t. Comrat, v. Lozova and v. Palanca. The highest numbers of specimens are assigned to Chlorophorus varius with 5 specimens, followed by Chlorophorus figuratus with 3 specimens. A single specimen of Agapanthia lederi is kept in this collection.

\section{DISCUSSION}

Papers including Cerambycidae species collected in present-day Republic of Moldova have been published since 1910, but not all species are deposited in the museums. Unfortunately, in the studied museum collections, there are no voucher specimens of Cerambycidae species cited as being collected in Moldova by some authors such as CHYUBCHIK (2010), NeCULISEANU \& BABAN (2005), Medvedev \& Shapiro (1957) and Plavilshtshikov $(1915,1940)$.

The annotated list of longhorn beetles published by CHYUBCHIK (2010) contains data about specimens of Cerambycidae collected in Central Moldova. A total of 78 species were cited, seven of them - Akimerus schaefferi (Laicharting, 1784), Arhopalus rusticus (Linnaeus, 1758), Asemum striatum (Linnaeus, 1758), Trichoferus campestris (Falderman, 1835), T. pallidus (Olivier, 1790), Callimoxys gracilis (Brullé, 1832) and Theophilea subcylindricolis Hladil, 1988 - are not present in the studied collections. These materials are kept in 13 private collections in Russia, Ukraine and the Republic of Moldova (Сну Uвснік 2010). 
According to the previously published checklist of Coleoptera from the Republic of Moldova (BACAL et al. 2013), 125 species of Cerambycidae were cited. In the checklist 26 species of Cerambycidae are mentioned as previously reported from the Republic of Moldova: Acanthocinus aedilis (Linnaeus, 1758), Acanthoderes clavipes (Scharnk, 1781), Agapanthia maculicornis (Gyllenhal, 1817), Cerambyx miles Bonelli, 1812, Clytus arietis (Linnaeus, 1758), C. tropicus (Panzer, 1795), Cyrtoclytus capra (Germar, 1824), Dorcadion aethiops (Scopoli, 1763), D. carinatum (Pallas, 1771), D. litiosum Ganglbauer, 1884, D. scopolii (Herbst, 1784), Pachyta quadrimaculata (Linnaeus, 1758), Pidonia lurida (Fabricius, 1792), P. suturalis Le Conte, 1858, Lamia textor (Linnaeus, 1758), Leptura bisignata Brullé, 1832 (Vadonia bipunctata globicollis (Desbrochers des Loges, 1870)), Molorchus umbellatarum (Schreber, 1759), Monochamus sutor (Fabricius, 1787), Phytoecia rubropunctata Goeze, 1777, P. scutellata (Fabricius, 1793), Phymatodes pusillus (Fabricius, 1787), Ropalopus femoratus (Linnaeus, 1758), R. varini (Bedel, 1870), Saperda carcharias (Linnaeus, 1758), Tetrops starki Chevrolat, 1859 and Xylotrechus pantherinus (Savenius, 1825). Unfortunately, there are no voucher specimens of these species in the collections of studied museums, and six specimens remained unidentified at species level.

In the monograph of DANILEVSKY (2014) other two species, Cortodera moldovana Danilevsky, 1996 and Grammoptera ustulata (Schaller, 1783) are mentioned. C. moldovana was described as endemic of Moldova, and the second species was collected in Cojusna locality, at 16 May 2009 by A. Zubov. Unfortunately, there are no specimens of these species in the studied museums (DANILEVSKy 2014).

Certain species of Cerambycidae are protected by European conventions (Annex II of the Bern Convention) and are included in the Red Books of several countries. In the Red Book of the Republic of Moldova (CARTEa RoşıE A Republicir Moldova 2015) six species, Aromia moschata, Cerambyx cerdo, Dorcadion equestre, Morimus funereus, Purpuricenus kaehleri and Rosalia alpina were included. Three of them, Rosalia alpina, Cerambyx cerdo and Morimus funereus are protected also through the European Union/Council of Europe Joint Programme „Emerald Network of Nature Protection Sites” at pan-European level.

On 8 May 2008, between localities Hânceşti and Rusca in Hânceşti district, several individuals of Pilemia tigrina were observed and photographed by A. Csathó in the forest steppe flora. Three voucher specimens were collected and deposited in the Coleoptera Collection of the HNHM.

The finding was published by Csathó (2014) as the first record for the country; however, the species was mentioned earlier from the Republic of Moldova by Miller \& Zubowsky (1917) and Medvedev \& Shapiro (1957). The correction was included in Tó тн et al. (2016).

In order to protect rare species, further specimens were not collected and deposited in museums. Observed species were only photographed and published (BUŞMACHIU 2019). 
The European Red List of saproxylic beetles contains 153 species within the subfamilies Cerambycinae and Prioninae native to or naturalized in Europe before AD 1500 (Nieto \& AleXANDER 2010). From the Cerambycidae known to occur in the Republic of Moldova, 48 species are included in the Red List. Of them, 44 are categorised as Least Concern (LC), three as Not Threatened (NT), and one as Data Deficient (DD).

Acknowledgments - The authors would like to thank all curators of the entomological collections: M. Batco (IGPP) and S. Poznacomchin (MSUM). The study was supported by the project "Evolutionary changes of economically important terrestrial fauna, of rare and protected species in the conditions of anthropic and climatic changes" (N. 20.80009.7007.02).

\section{REFERENCES}

Althoff J. \& Danilevsky M. 1997: A check-list of Longicorn beetles (Coleoptera, Cerambycoidea) of Europe. - Slovensko Entomolosko Drustvo Stefana Michielija, Ljubljana, 64 pp.

Bacal S., Munteanu N. \& Toderaş I. 2013: Checklist of beetles (Insecta: Coleoptera) of the Republic of Moldova. - Brukenthal Acta Musei 8(3): 415-150.

BUŞMACHIU G. 2019: New record of some rare and protected insects species from the Republic of Moldova. - Marisia. Studii şi materiale. Ştiințele Naturii 38-39: 35-42.

Cartea Roşie a Republici Moldova 2015: Cartea Roşie a Republicii Moldova. Ediția a treia. The Red Book of the Republic of Moldova. Third edition. - Editura Ştiința, Chişinău, 492 pp.

Cнучвснік V. 2010: The annotated list of longicorn beetles (Coleoptera: Cerambicydae) of

Central Moldova. - Russian Entomological Journal 19(2): 111-118.

https://doi.org/10.15298/rusentj.19.2.04

Csatнó A. I. 2014: Pilemia tigrina (Mulsant, 1851), new for the fauna of Republic of Moldova

(Coleoptera: Cerambycidae). - Folia entomologica hungarica 75: 69-72.

https://doi.org/10.17112/FoliaEntHung.2014.75.69

Danilevsky M. L. 2014: Жужи-усачи (Coleoptera, Cerambycoidea) России и соседних стран.

Yacms 1. (Longicorn beetles (Coleoptera, Cerambycoidea) of Russia and adjacent countries.

Part 1.) - Higher School Consulting, Moscow, 518 pp.

Danilevsky M. L. 2019: Catalogue of Palaearctic Cerambycoidea. - Online:

www.zin.ru/Animalia/ Coleoptera/rus/danlists.htm [Accessed 23 August 2020.]

Derjanschi V., Baban E., Calestru L., Stahi N. \& Țugulea C. 2016: Catalogue of the "N. Zubowsky entomological collection". - Academy of Sciences of Moldova, National Museum of Ethnography and Natural History, Institute of Zoology, Chişinău, 296 pp. 
Medvedev S. \& Shapiro D. 1957 = Meabeaeb C. И. \& ШАПиро А. С. 1957: К познанию фауны жуков (Coleoptera) Молдавской ССР и сопредельных районов Украины. [To the knowledge of the beetle fauna (Coleoptera) of the Moldavian Soviet Socialist Republic and the neighbouring districts of Ukraine.] - Труды научно-исследовательского института биологии и биологического факультета Харьковского Государственного Университета [Scientific works of the biological institute and biological department of the Kharkov State University] 30: 173-206.

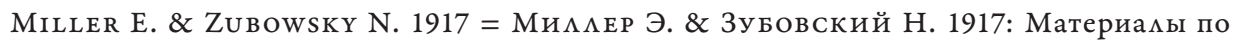
энтомологической фауне Бессарабии. [Materials on the entomological fauna of Bessarabia.] - Труды Бессарабского Общества Естествоиспытателей и Аюбителей Eстествознания [Proceedings of the Bessarabian Society of Naturalists and Life Science Amateurs] 6: 119-150.

NeARns E. H. 2013: Systematics of Longhorned Beetles (Insecta: Coleoptera: Cerambycidae). The University of New Mexico, Digital repository. Online:

https://digitalrepository.unm.edu/biol_etds/86 [Accessed 23 August 2020.]

Neculiseanu Z. \& Baban E. 2005: Fauna cerambicidelor (Coleoptera, Cerambycidae) din Republica Moldova. - Analele Ştiințifice ale Universității de Stat din Moldova 2005: 199-202.

Nieto A. \& Alexander K. N. A. 2010: European Red List of Saproxylic Beetles. - Publications Office of the European Union, Luxembourg, VIII+45 pp.

Plavilshtshikov N. N. 1915 = Пцавицьщиков Н. Н. 1915: Палеарктические виды рода Rhagium F. (Coleoptera, Cerambycidae). (Les espèces paléarctiques du genre Rhagium F. (Coleoptera, Cerambycidae).) - Русское энтомологическое обозрение (Revие russe d'Entomologie) 15(1): 32-49.

Plavilshtshikov N. N. $1940=$ Плавимьщиков Н. Н. 1940: Фауна СССР. Насекомые. Жесткокрылые. Том XXII, вып. 2. Жуки-дровосеки. Часть 2. [Fauna of URSS. Insects. Beetles. Volume 22, issue 2. Cerambycidae. Part 2.]. - ИзАательство Академии Наук CCCP, Москва- $\Lambda$ енинграА [Publishing house of the Academy of Sciences of the USSR, Moscow-Leningrad], $785 \mathrm{pp}$.

Plavilshtshikov N. N. 1958 = Плавильщиков Н. Н. 1958. Фауна СССР. Жесткокрылые. Том XXIII, вып. 1. Жуки-дровосеки. Часть 3. Подсемейство Lamiinae, ч. 1. [Fauna of URSS. Beetles. Volume 23, issue 1. Cerambycidae. Part 3. Subfamily Lamiinae, part 1.]. Издательство Академии Наук СССР, Москва-Аенинград [Publishing House of the Academy of Sciences of the USSR, Moscow-Leningrad], $592 \mathrm{pp}$.

Plavilshtshikov N. N. $1965=$ П Аавимьщиков Н. Н. 1965: 75. Сем. Cerambycidae - ЖукиАровосеки, усачи. [75th fam. Cerambycidae - Longicorn beetles.] - In: ГУрьева Е. $\Lambda$. \& Крыжановский О. $\Lambda$. (eds): Определитель насекомых Европейской части СССР в плти томах. II. Жесткокрылые и веерокрылые. [Identification key to the insects of the European part of the USSR in five volumes. II. Coleoptera and Strepsiptera. ]. ИзАательство

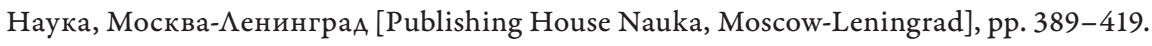

Tóth I. Z., Csathó A. I., Buşmachiu G. \& Merkl O. 2016: Pilemia tigrina: new and corrected records from the Republic of Moldova, Hungary and Romania (Coleoptera: Cerambycidae). Folia entomologica hungarica 77: 33-40.

https://doi.org/10.17112/FoliaEntHung.2016.77.33 